NISTIR 6705

\title{
A Sensor-Driven Fire Model Version 1.1
}

William D. Davis

Glenn P. Forney

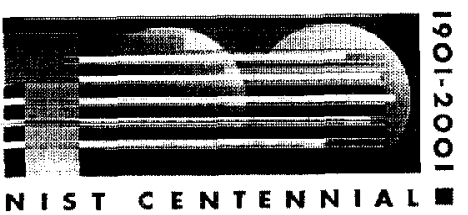

NLT

National Institute of Standards and Technology Technology Administration, U.S. Department of Commerce 

NISTIR 6705

\title{
A Sensor-Driven Fire Model Version 1.1
}

\author{
William D. Davis \\ Glenn P. Forney \\ Building and Fire Research Laboratory \\ Gaithersburg, MD 20899-8642
}

January 2001

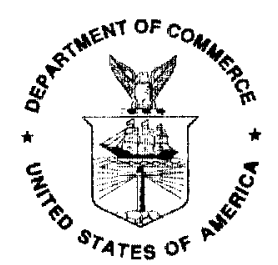

U.S. Department of Commerce Norman Y. Mineta, Secretary

Technology Administration Dr. Cheryl L. Shavers, Under Secretary of Commerce for Technology

National Institute of Standards and Technology Karen H. Brown, Acting Director 


\section{CONTENTS}

Page

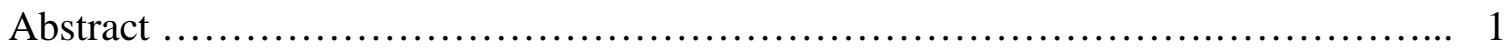

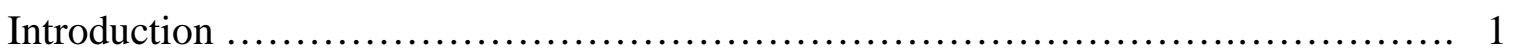

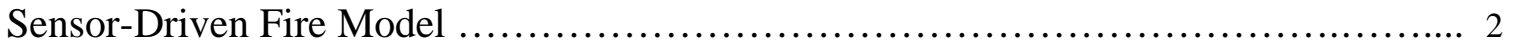

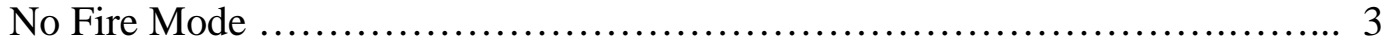

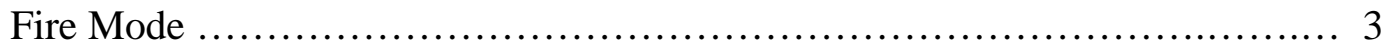

HRR from ceiling jet temperature ...................................... 4

HRR from ceiling jet smoke concentration $\ldots \ldots \ldots \ldots \ldots \ldots \ldots \ldots \ldots \ldots \ldots \ldots, 6$

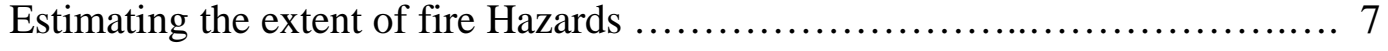

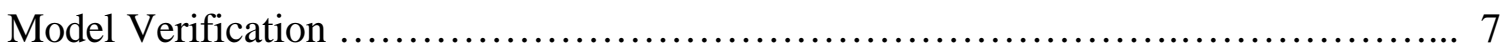

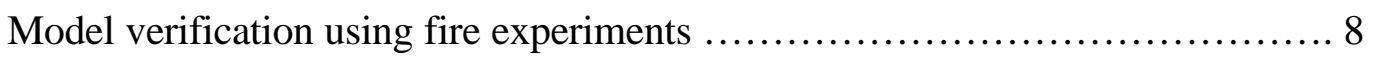

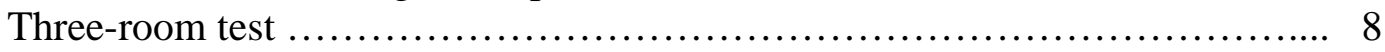

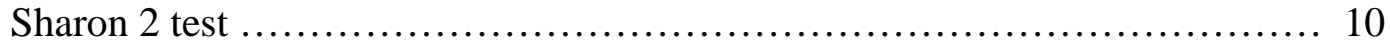

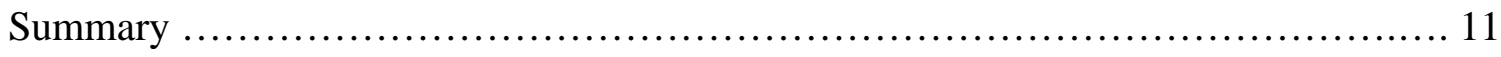

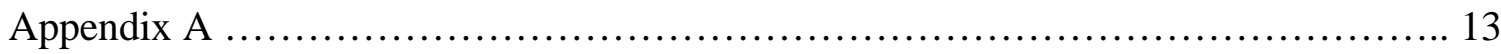

Sensor-driven model ................................................... 13

Zone model ............................................................ 15

Appendix B .......................................................... 17

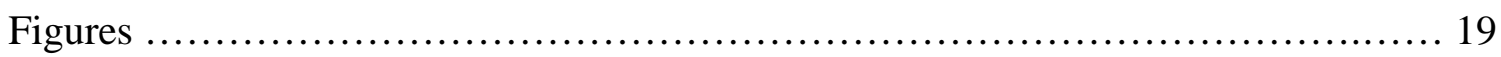

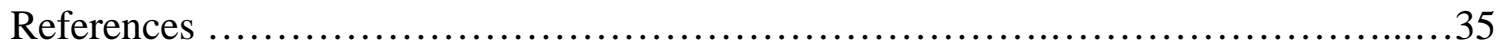





\title{
A Sensor-Driven Fire Model \\ Version 1.1
}

\author{
William D. Davis \& Glenn P. Forney
}

\begin{abstract}
Modern building fire sensors are capable of supplying substantially more information to the fire service than just the simple detection of a possible fire. With the increase in the number of sensors installed in buildings for non-fire purposes, it is possible to capture this diverse information as input to fire alarm systems to enhance the value of the information in both fire and non-fire conditions. In order to use this information, a fire model needs to be developed that interprets a range of sensor signals and provides information about the building environment to the fire panel. Typical fire models useful for predicting the impact of fire in a building utilize a prescribed heat release rate (HRR) for the fire and can predict sensor response. For the inverse problem, a sensor-driven fire model uses sensor signals to estimate the HRR of the fire, identify areas where hazardous conditions are developing, and predict the development of the fire.
\end{abstract}

A sensor-driven fire model (SDFM) is being developed at NIST for the NIST Virtual Cybernetic Building Test-bed to investigate the feasibility of such a model in buildings with HVAC systems. Version 1.1 of SDFM uses ceiling jet algorithms for temperature and smoke concentration to convert the analog or digital data from heat and smoke detectors to a HRR. A version of CFAST is then used to obtain layer temperatures and depths for the room of fire origin as well as surrounding rooms. With this information, the growth and spread of the fire and the location of hazardous conditions can be estimated. Details of SDFM will be presented and comparisons with experiments will be provided.

\section{Introduction}

Modern building fire sensors are capable of supplying substantially more information to the fire service than just the simple detection of a possible fire. Nelson, in 1984, recognized the importance of tying all the building sensors to a smart fire panel. ${ }^{i}$. In this report, a sensor-driven fire model (SDFM) is described that is designed to achieve a smart fire panel configuration such as envisioned by Nelson. A sensor-driven fire model makes use of signals from a variety of detectors such as smoke, heat, gas, etc. to detect, verify and predict the evolution of a fire in a building. In order to accomplish this task, the fire model must be able to discriminate between fire and non-fire conditions, must be able to recognize detector failure for both fire and non-fire scenarios, and must be able to determine the size, location, and potential hazards associated with a growing fire.

The fire model must be flexible by having the capability to handle fire scenarios in rooms where there may be anywhere from a suite of detectors to no detectors. In the latter case, detectors in adjacent rooms would provide the necessary sensor input to the model. For the suite of detectors, the model must be able to take advantage of the increased amount 
of information in order to provide earlier and more reliable detection. The model must also be able to accommodate detector failures due to a growing fire and still continue to provide estimations of fire growth and location. Finally, the model must be able to handle a large number of rooms and must complete its calculation cycle in a time interval that is shorter than real time.

The sensor-driven fire model described in this paper is at an early stage of development with only a subset of the required features present. In the next sections of this paper, SDFM will be described and comparisons with experiments will be presented.

\section{Sensor-Driven Fire Model}

Version 1.1 of SDFM is designed to predict the heat release rate (HRR) of a fire based on signals from either smoke or heat detectors positioned below the ceiling that sample the ceiling jet produced by the fire. The estimated HRR is then used by a variant of the zone model CFAST to predict layer temperatures and heights in the fire room and in the adjacent rooms in the building. Based on the predicted layer temperatures and height, room conditions such as limited visibility and flashover potential can be deduced.

In non-fire situations, the SDFM is designed to look for sensor failure, to discriminate between nuisance signals and fire induced signals, and to monitor the condition of detectors that degrade over time.

The structure of SDFM is as follows:

1. The user enters building information that includes the number, location, size, type of vents, and connectivity of the rooms, corridors and other spaces.

2. The user enters the location, type, calibration constants, and set point for each detector.

3. SDFM initializes itself and begins to learn about the building environment.

4. SDFM routinely cycles through all the building detectors checking for signs of sensor failure or possible hazardous/fire conditions.

5. The user will be notified of the location and type of each detector that is identified as having failed or otherwise in need of replacement.

6. If SDFM identifies a possible fire signal, a false alarm protocol will be used to verify that the sensor signal is responding to a real fire.

7. If the possible fire signal verifies as a real fire, the model will estimate fire size and location, predict fire growth and spread, and identify possible fire associated hazards in rooms with or without fire.

Details of the structure of the SDFM will be described in subsequent sections. A simple flowchart of the SDFM is provided in figure 1. 


\section{No Fire Mode}

SDFM will spend virtually all its time monitoring the building detectors in the no fire mode. In this mode, the signal received from each detector will be compared with the historic detector record to identify any deviation from normal operation. Detector failure modes will be checked and sensor signals that fall into these modes will result in a trouble (sensor failure) signal being sent to the appropriate monitoring location.

Version 1.1 of SDFM has only a simple checking algorithm available that detects sensor failure based on either no signal or a saturated signal from the detector. As this section of SDFM is expanded, it is planned to allow a user to enter failure modes that are particular to a specific detector.

\section{Fire Mode}

When SDFM receives a detector signal that indicates a HRR increasing with time and has reached a target threshold, SDFM will try to verify that it is a true fire by assessing the signals received by other available detectors in the area. Such detectors might include $\mathrm{CO}$ or $\mathrm{CO}_{2}$ detectors as well as heat or smoke detectors. If other detectors do not support the fire signal, a trouble signal will be issued and the program will revert to its normal detector polling. If no other detectors are available in the room or if other sensors also support the presence of a fire, a fire alarm will be issued.

The target threshold for SDFM to start checking for a fire is based on two alternative methods of defining a fire signal. The first method used by SDFM is to compare the sensor signal with a user prescribed signal. This signal would be one that has been developed by observing the response of the detector to small test fires. The second method would be based on looking at the time history of the detector signal once an estimated HRR based on the detector signal has been reached. If the detector signal indicates a time growing hazard that has reached a particular HRR, a fire alarm will be issued. This second method may allow for earlier detection of fires as well as fewer false alarms since it depends on a time history as well as a signal magnitude.

The determination of a HRR from a detector signal requires knowledge about the characteristics of the detector and its position with respect to the fire. Detector characteristics include the calibration curve for the analog/digital signal generated by the detector as a function of temperature or smoke/gas concentration and the delay time introduced by thermal lag or flow conditions into the sensing element. Once the detector characteristics have been defined, the HRR may be estimated using modeling correlations coupled with a zone fire model. In the following discussion, it will be assumed that only one detector is present in each room. The detector will be located close to the ceiling where it can be considered in the ceiling jet. Presently, version 1.1 of SDFM contains algorithms to estimate HRR from either the excess temperature or the smoke concentration in the ceiling jet. 


\section{HRR from ceiling jet temperature}

The algorithm used in version 1.1 of SDFM to determine the HRR from the ceiling jet temperature was developed by Davis ${ }^{\mathrm{ii}}$. The algorithm gives the ceiling jet temperature as a function of radial distance from the plume centerline as a function of HRR, layer depth and temperature, and location of the fire. When the layer depth is less than 0.05 of the fire to ceiling height, it is assumed that a layer has developed and the radial dependence of the temperature of the ceiling jet falls off as $r^{-.23}$. The layer is assumed to be sufficiently thin that the plume centerline temperature is not increased by the presence of the layer. With the above assumptions, the convective HRR can be determined from

$$
Q_{c}=0.172 H_{1}^{5 / 2}\left(\frac{r}{0.18 H_{1}}\right)^{0.345}\left(\frac{\Delta T_{c j}^{3 / 2}}{T_{\infty}^{1 / 2}}\right)
$$

$\mathrm{Q}_{\mathrm{c}}$ is the convective heat release rate, $\mathrm{H}_{1}$ is the ceiling height above the fire surface, $\mathrm{r}$ is the radial distance from the plume centerline, $\Delta \mathrm{T}_{\mathrm{cj}}$ is the excess ceiling jet temperature, and $\mathrm{T}_{\infty}$ is the ambient temperature. To get the total heat release rate, the radiative fraction of the fuel, $\chi_{\mathrm{r}}$, must be estimated. For large fires, the radiative fraction will be relatively small, perhaps on the order of $0.2^{\mathrm{iii}}$. The total heat release rate, $\mathrm{Q}$, would then be given by

$$
Q=\frac{Q_{c}}{\left(1-\chi_{r}\right)}
$$

When the layer depth exceeds 0.05 of the fire to ceiling height, $\mathrm{Q}_{\mathrm{c}}$ is obtained in the following manner. First, the plume centerline temperature excess, $\Delta \mathrm{T}_{\mathrm{p}}$ is estimated using the ceiling jet correlation of Davis ${ }^{2}$, assuming that the layer is sufficiently deep that the correlation becomes

$\Delta T_{p}=1.19\left(\Delta T_{c j}\right)\left(\frac{r}{.18 H_{1}}\right)^{.23}$

The method of Evans ${ }^{\text {iv }}$ is then used to estimate $Q_{c}$ based on the estimated plume centerline temperature. The equation used for predicting the plume centerline temperature assuming a single upper layer with temperature $\mathrm{T}_{\mathrm{u}}$, fire to ceiling height $\mathrm{H}_{2}$, and dimensionless virtual source strength at the layer interface $\mathrm{Q}_{1,2}$ 
$\Delta T_{p}=9.28 T_{u} Q_{I, 2}^{2 / 3}\left(\frac{z_{I, 2}}{H_{2}}\right)^{5 / 3}$

is combined with the equations relating the ratio of the distance from the fire to the layer interface for the actual and virtual fire sources, $\mathrm{z}_{\mathrm{I}, 1}$ and $\mathrm{z}_{\mathrm{I}, 2}$

$\frac{z_{I, 2}}{z_{I, 1}}=\left[\frac{\xi Q_{I, 1} C_{T}}{Q_{I, 2}^{1 / 3}\left[(\xi-1)\left(\beta^{2}+1\right)\right]+\xi C_{T} Q_{I, 2}^{2 / 3}}\right]^{2 / 5}$

and the equation which gives the distance of the virtual source to the ceiling

$H_{2}=H_{1}-z_{I, 1}+z_{I, 2}$

to yield an equation for the strength of the virtual source at the layer interface, $\mathrm{Q}_{\mathrm{I}, 2}$. $\xi$ is the ratio of the upper layer to lower layer temperature, $\beta$ is the velocity to temperature ratio of Gaussian profile half widths (.9555) and $C_{T}=9.115$. The resulting equation is solved using a root finding algorithm.

$$
f\left(Q_{I, 2}\right)=\frac{\frac{H_{1}}{z_{I, 1}}-1}{\left[\frac{\xi C_{T}\left(\frac{\xi C_{T} Q_{I, 2}^{2 / 3}+\xi-1}{C_{T}}\right)^{3 / 2}}{Q_{I, 2}^{1 / 3}\left[(\xi-1)\left(\beta^{2}+1\right)+\xi C_{T} Q_{I, 2}^{2 / 3}\right]}\right]^{2 / 5}}+1-\frac{Q_{I, 2}^{2 / 5}}{\left[\frac{\Delta T_{P}}{9.28 T_{u}}\right]^{3 / 5}}
$$

The source strength at the layer interface is found using

$Q_{I, 1}=\left[\frac{\xi C_{T} Q_{I, 2}^{2 / 3}+\xi-1}{C_{T}}\right]^{3 / 2}$

The convective heat release rate of the fire is calculated using

$Q_{c}=Q_{I, 1} \rho_{\infty} c_{\infty} T_{\infty} g^{1 / 2} z_{I, 1}^{5 / 2}$ 
For the case when the layer depth is less than 0.05 of the fire to ceiling height, the values of four unknowns, $\mathrm{H}_{1}, \chi_{\mathrm{r}}, \mathrm{r}$, and $\Delta \mathrm{T}_{\mathrm{cj}}$ must be determined. When the layer exceeds 0.05 of the fire surface to ceiling height, five unknowns, $\mathrm{H}_{1}, \chi_{\mathrm{r}}, \mathrm{r}, \Delta \mathrm{T}_{\mathrm{cj}}$ and $\mathrm{T}_{\mathrm{u}}$ must be evaluated. The value of $\Delta \mathrm{T}_{\mathrm{cj}}$ is measured using heat sensors in the ceiling jet. The value of $\chi_{\mathrm{r}}$ will be assumed to be 0.3 , a reasonable value for large fires.

In situations where only one heat detector is available in the room of fire origin, the values of $\mathrm{H}_{1}$ and $\mathrm{r}$ must be assumed in order to obtain a crude estimate of the HRR. A reasonable estimate for enclosures of normal room height would be that the ceiling height above the fire surface, $\mathrm{H}_{1}$, is approximately $0.75 \mathrm{H}_{\mathrm{r}}$, where $\mathrm{H}_{\mathrm{r}}$ is the floor to ceiling height. For rooms where high rack storage exists, a smaller value may be warranted. The value for $r$ is estimated as equal to 0.5 times the maximum length from the detector to a room wall. Since the radial dependence is not strong, this value is not nearly as important to the calculation as the estimate for $\mathrm{H}_{1}$.

For two detectors present in the room, an estimate for $\mathrm{H}_{1}$ still needs to be made. The estimate for $\mathrm{H}_{1}$ of $0.75 \mathrm{H}_{\mathrm{r}}$ is still appropriate but the radial distance to the fire can now be estimated if the detector spacing in the room is known. As additional detectors at different locations in the room become available, timing measurements can be used to estimate the value for $\mathrm{H}_{1}$ while the value of $\mathrm{r}$ may be deduced using the ceiling jet correlation.

The upper layer temperature $\mathrm{T}_{\mathrm{u}}$ and depth is calculated using the zone model once a HRR and $\mathrm{H}_{1}$ have been determined. The upper layer temperature and depth are used as inputs when the next iteration of detector signals are sent to the SDFM.

\section{HRR from ceiling jet smoke concentration}

An algorithm has been developed which provides an estimate of the maximum smoke mass concentration in the ceiling jet as a function of position and $\mathrm{HRR}^{\mathrm{v}}$. The maximum smoke mass concentration in the ceiling jet is given by

$$
C_{s 0}(r)=\frac{7.94 Y_{s} Q^{2 / 3}\left(\frac{H_{1}}{r}\right)^{0.57}}{h_{c}\left(1-\chi_{r}\right)^{1 / 3}\left(1+\frac{0.0853\left(1-\chi_{r}\right)^{2 / 3} Q^{2 / 3}}{\left(H_{1}-z_{0}\right)^{5 / 3}}\right)\left(H_{1}-z_{0}\right)^{5 / 3}}
$$

where $\mathrm{C}_{\mathrm{s} 0}$ is the maximum smoke mass concentration in the ceiling jet, $\mathrm{r}$ is the distance from the plume centerline, $\mathrm{H}_{1}$ is the distance from the fire surface to the ceiling, $\mathrm{h}_{\mathrm{c}}$ is the 
heat of combustion, $\mathrm{Y}_{\mathrm{s}}$ is the smoke yield fraction, $\chi_{\mathrm{r}}$ is the radiative fraction, $\mathrm{Q}$ is the total heat release rate, $\mathrm{z}_{0}$ is the virtual origin ${ }^{\mathrm{vi}}$. This equation is inverted in the SDFM such that the HRR is calculated from the measured smoke mass concentration. This correlation was developed for the unconfined ceiling situation and must be modified once a smoke layer develops. In version 1.1 of the SDFM, no modification for the impact of a smoke layer is calculated which means that this algorithm will provide an overestimate of the HRR.

\section{Estimating the extent of fire hazards}

Once a HRR has been obtained for one or more of the identified fire sources, this information will be passed to a version of CFAST in order to calculate layer height, temperatures and smoke concentrations in each room of the structure. From this information, hazards such as limited sight, high temperatures and potential for flashover may be identified on a room by room basis for the current fire conditions.

The layer temperatures and smoke concentrations calculated using CFAST are also used to estimate fire spread from the room of origin to adjacent rooms. The signals from sensors in these adjacent rooms are compared with calculated signals based on the estimated layer temperature and smoke concentration predicted by CFAST. If the ceiling jet temperature as estimated from sensor signals is $30 \%$ higher than the upper layer temperature predicted by CFAST, it is assumed that a fire has broken out in the adjacent room. If the predicted upper layer temperature exceeds the flashover temperature, 500 ${ }^{\circ} \mathrm{C}$, it is assumed that a fire has started in that room.

In addition, with a known HRR history, projections can be made using CFAST to estimate fire growth and spread. The present version of SDFM does not have this capability.

\section{Model Verification}

Since the SDFM is designed to operate in a space with a large number of rooms, verification of the algorithms becomes a major challenge. One method of verification will be to use the results of multiple room fire experiments and to test the predictions of the SDFM against these experiments. The number of these fire experiments is quite limited, so an additional method of verification is being used. The Virtual Cybernetic Building Testbed at NIST is a computer platform where the building ventilation, heating and cooling, and sensor activities in a multiple room building can be simulated. The present structure modeled in the test-bed contains three rooms and will soon be increased to nine rooms. Using CFAST ${ }^{\mathrm{vii}}$ or FDS ${ }^{\mathrm{viii}}$, a fire scenario can be generated for the testbed room geometries and the resulting sensor signals used as the model input for the SDFM. In this way, the SDFM can be tested using a virtual fire source and receive signals which are representative of what may happen in an actual fire scenario in the virtual building. 
An example of a simulation of a fire scenario for the three-room test-bed using the visualization software, NIST Smokeview ${ }^{\mathrm{ix}}$, is shown in figure 2. A fire starts in room 1 and grows rapidly with the SDFM issuing warnings of a growing fire and showing the present room conditions for limited sight, toxic gas/thermal hazard or flashover. As fires start in the other rooms, the SDFM identifies the fire condition and ends up following a multiple room, multiple fire scenario.

The SDFM has been tested with scenarios that had as many as fourteen rooms with four of the rooms containing fires. The calculation cycle for SDFM has been significantly faster than real time for these scenarios.

\section{Model verification using fire experiments}

Two fire experiments were selected to provide initial verification of the SDFM. The first is a three room, single level experiment where the fire source was a methane burner ${ }^{\mathrm{X}}$. The second experiment is a seven room, two story full scale fire test (Sharon 2) where the major fuel source was wood pallets and flashover was achieved in the burn room ${ }^{\mathrm{xi}}$. Thermocouple data was available for both experiments and single thermocouples near the ceiling were used for the SDFM inputs to mimic the response of ceiling mounted heat detectors

\section{Three-room test}

A plan view of the test is shown in figure 3. The test was modeled as a three-room structure with two short corridors connecting the rooms being modeled as doors. The exit door to the outside was assumed to empty into a large (ambient) room with no air outflow. Since an exhaust hood was positioned above this exit, there was flow present but the amount of flow exiting the experiment was not measured and hence was not modeled. While multiple thermocouple trees and sensors were available in each room, only one thermocouple per room was used to provide a sparse data set for comparison with room conditions. A sparse data set does not provide sufficient information to locate the fire and therefore provides a test of the default positioning algorithm used in the SDFM.

The experiment consisted of a methane burner operating at $2.8 \mathrm{~kW}$ for the first $330 \mathrm{~s}$ of the test. The $2.8 \mathrm{~kW}$ fire was increased to $103 \mathrm{~kW}$ at $340 \mathrm{~s}$ into the test. The SDFM detected a fire in the burn room at $350 \mathrm{~s}$ into the test. The initial fire of $2.8 \mathrm{~kW}$ was below the threshold setting for fire detection in the SDFM.

Figure 4 provides a comparison of the predicted upper layer temperatures of the SDFM with the calculated upper layer temperatures of the experiment. The plots start from time 0 that is the time that the SDFM detected a fire (350 s into the experiment). " 1 exp" is the first room experimental results while "1 SDFM" is the first room SDFM prediction. The SDFM predictions are higher than the calculated upper layer temperatures of the experiment for the second room but are in good agreement for rooms one and three. The 
average $95 \%$ confidence interval for this data based on five identical experiments is \pm 18 ${ }^{\circ} \mathrm{C}, \pm 6{ }^{\circ} \mathrm{C}$, and $\pm 4{ }^{\circ} \mathrm{C}$ for rooms 1,2 , and 3 respectively.

The layer height comparisons for room 2 are given in figure 5. The experimentally measured layer heights are based on observations of the height of the smoke layer in the experiment and are not inferred from temperature measurements. Agreement between the observed layer heights and calculated layer heights are quite good. The average $95 \%$ confidence interval for this measurement is $\pm 0.2 \mathrm{~m}$.

The SDFM is designed to provide information concerning the fire threat that fire fighters might encounter in a building. The fire threats presently in the SDFM include: a smoke layer less than $2 \mathrm{~m}$ above the floor (limited visibility), a smoke layer above a temperature of $50{ }^{\circ} \mathrm{C}$ and layer height below $1.5 \mathrm{~m}$ (toxic gas/thermal hazard), and a smoke layer at a temperature higher than $500{ }^{\circ} \mathrm{C}$ (flashover). For the three-room experiment, the upper layer temperature did not reach flashover and no flashover warnings were issued. A comparison of the SDFM predictions with the experimental measurements is given in the table below. The SDFM was run with a reporting interval of $20 \mathrm{~s}$.

\begin{tabular}{|l|l|l|l|l|l|l|}
\hline Room & 1 exp. & 1 SDFM & 2 exp. & 2 SDFM & 3 exp. & 3 SDFM \\
\hline Visibility Limited & na & $20 \mathrm{~s}$ & $20 \mathrm{~s}$ & $40 \mathrm{~s}$ & na & $60 \mathrm{~s}$ \\
\hline $\begin{array}{l}\text { Toxic Gas/Thermal } \\
\text { Hazard; Layer }\end{array}$ & na & $100 \mathrm{~s}$ & $190 \mathrm{~s}$ & $100 \mathrm{~s}$ & na & $\mathrm{nr}$ \\
\hline $\begin{array}{l}\text { Toxic Gas/Thermal } \\
\text { Hazard; Temperature }\end{array}$ & $0 \mathrm{~s}$ & $20 \mathrm{~s}$ & $50 \mathrm{~s}$ & $100 \mathrm{~s}$ & $460 \mathrm{~s}$ & $80 \mathrm{~s}$ \\
\hline
\end{tabular}

Table 1 Comparison of time of occurrence for three hazard predictions. The symbol "na" stands for not available and "nr" is not reached. 1 exp is the first room experimental results while $1 \mathrm{SDFM}$ is the first room SDFM prediction.

Experimental layer heights were given for only room 2 in the experiment. Hence, the toxic gas/thermal hazard warning has been separated into two parts, one the smoke layer temperature in excess of $50{ }^{\circ} \mathrm{C}$ and the other for a layer height, $<1.5 \mathrm{~m}$. In room 1 , the SDFM provided a toxic gas/thermal hazard warning as quickly as it could (based on temperature) and is in good agreement with the experimental measurement.

In room 2, the limited visibility warning was in good agreement with the experimental measurements. The toxic gas/thermal hazard warning was issued roughly $90 \mathrm{~s}$ ahead of the criteria being met experimentally. This difference is due to the experimental layer height staying just above the $1.5 \mathrm{~m}$ layer height criterion while the calculation predicted a layer height that is just below the $1.5 \mathrm{~m}$ criterion (see figure 5).

In room 3, the layer temperature in the experiment remains just below the $50{ }^{\circ} \mathrm{C}$ criterion while the calculated value is just above the $50{ }^{\circ} \mathrm{C}$ criterion (see figure 4 ) for an extended time period. The small differences in the experimental and calculated temperatures account for the large difference in the time to issue the warning. 
Based on the results for rooms 2 and 3, the warning levels used to issue hazard warnings should be set at a lower value than the hazard level so that small differences in the calculations will not delay potential hazard warnings.

\section{Sharon 2 test.}

To simulate the Sharon 2 test, the seven-room townhouse was divided into eight spaces and thermocouple data was used to provide ceiling jet temperatures in six of the eight spaces. A plan view of the townhouse, showing locations of the instrumentation, is shown in figure 6 . In the simulation, room 2 was partitioned into a hallway and a room with the thermocouple tree, TC6, providing the temperatures for the hallway. Thermocouple trees TC1, TC3, and TC4 were used to provide data for the upstairs spaces while TC8 and TC1 were used for the other downstairs spaces. The partitioned room 2 on the first floor and the stairway were modeled spaces with no thermocouple measurements. Only the thermocouple near the ceiling was used for the input data from each tree. The object was to simulate the data from a single, spot-type heat detector in a room. Only one detector per room was used in order to see how SDFM would perform with a sparse data set. In the sparse data set algorithm, the location of the fire must be assumed which will provide only approximate information on room conditions.

The first 231 seconds of the fire was modeled for the Sharon 2 fire since, in the experiment the wood pallets in the fire room began to fall off the load cell after this time. Figures 7-11 provides a comparison of a representative layer temperature as measured by the thermocouple trees with the layer temperature calculated by SDFM for each room. Agreement between the calculations and the measurements is quite good for all rooms although SDFM tends to overpredict the temperature.

Figure 7 is particularly instructive in that it demonstrates the feedback mechanism that is contained in SDFM. The SDFM detected the fire $36 \mathrm{~s}$ after the fire started which caused it to overpredict the HRR based on sensor temperature due to the under estimated temperature and depth of the upper layer. As a result, the upper layer temperature was overpredicted, but as SDFM acquired more data measurements, the HRR was corrected to yield the appropriate upper layer temperature. At about $100 \mathrm{~s}$ after the SDFM began its calculations, an additional ventilation source was opened which resulted in an increase in temperature of the upper layer. The SDFM again initially overpredicted the upper layer temperature but with increasing numbers of data points, the correct upper layer temperature was obtained. The feedback mechanism contained in SDFM appears to work well and allows SDFM to follow changes in ventilation conditions for rooms containing the fire.

The experimental layer height was determined by estimating the location of the midpoint of the temperature transition between the lower layer temperature and the upper layer temperature. The location of the midpoint above the floor was taken as the upper layer height. Figures 12-16 provide a comparison of the estimated upper layer heights with the 
calculated values over a period of $195 \mathrm{~s}$ from the detection time of the fire. The fire actually started somewhat earlier but did not trigger the detection algorithm in the SDFM until about $36 \mathrm{~s}$ after the fire was ignited. The calculated layer heights agree well with the estimated layer heights for most of the comparison interval. Only at the last time intervals for the second floor bedrooms do the calculated upper layer heights drop significantly below the estimated layer heights.

The SDFM is designed to provide information concerning the fire threat that fire fighters will encounter in a building. The fire threats presently in SDFM include: a smoke layer less than $2 \mathrm{~m}$ above the floor (limited visibility), a smoke layer at a temperature of $50{ }^{\circ} \mathrm{C}$ and layer height at $1.5 \mathrm{~m}$ (toxic gas/thermal hazard), and a smoke layer at a temperature higher than $500{ }^{\circ} \mathrm{C}$ (flashover). The predicted fire threats correlated well with the estimated occurrence of these threats for the lower level rooms using the data shown in table 2 below. The layer heights were predicted to be lower for the upper level rooms than measured which accounts for most of the differences shown in the table for these rooms. For the bedrooms, the smoke in the room may become well mixed with a two layer structure beginning to disappear. The cycle time for the calculations and measurements was $12 \mathrm{~s}$, meaning those two or three calculations or measurement times produced several of the time differences.

\begin{tabular}{|l|l|l|l|l|l|l|l|l|l|l|}
\hline Room & $\begin{array}{l}\text { Burn } \\
\text { rm } \\
\text { exp }\end{array}$ & $\begin{array}{l}\text { Burn } \\
\text { rm } \\
\text { sdfm }\end{array}$ & $\begin{array}{l}\text { LL } \\
\text { Hall } \\
\text { exp }\end{array}$ & $\begin{array}{l}\text { LL } \\
\text { Hall } \\
\text { sdfm }\end{array}$ & $\begin{array}{l}\text { UL } \\
\text { Hall } \\
\text { exp }\end{array}$ & $\begin{array}{l}\text { UL } \\
\text { Hall } \\
\text { sdfm }\end{array}$ & $\begin{array}{l}\text { N } \\
\text { Rm } \\
\text { exp }\end{array}$ & $\begin{array}{l}\text { N } \\
\text { Rm } \\
\text { sdfm }\end{array}$ & $\begin{array}{l}\text { S } \\
\text { Rm } \\
\text { exp }\end{array}$ & $\begin{array}{l}\text { S } \\
\text { Rm } \\
\text { sdfm }\end{array}$ \\
\hline Visibility Limited & 24 & 12 & 49 & 12 & 88 & 24 & 78 & 49 & 80 & 36 \\
\hline $\begin{array}{l}\text { Toxic Gas/ } \\
\text { Thermal Hazard }\end{array}$ & 24 & 12 & 49 & 24 & 99 & 36 & 103 & 61 & 94 & 49 \\
\hline Flashover & 48 & 24 & 97 & 97 & nr & nr & nr & nr & nr & nr \\
\hline
\end{tabular}

Table 2 The table presents a comparison of the times in seconds between the experiment and the SDFM for the hazard conditions in the burn room, the lower level hall, the upper level hall and the two upper level bedrooms labeled $\mathrm{N}$ (north) and S (south). The symbol $\mathrm{nr}$ indicates that this condition was not reached.

\section{Summary}

The goal for the SDFM is to provide adequate warning of fire threats within a structure using the building sensors as detectors. For comparisons with two fire experiments, fire warnings were given that were in reasonable agreement with measurements. This agreement was obtained using data from only one sensor per room. Additional sensors in each room would permit the fire source to be more accurately located and as a result better predictive capabilities would be expected. The present results, using only single detectors in each room, provide information that would be of value to fire fighters.

The SDFM has a calculation cycle that is substantially faster than real time. A limit for how many rooms can be handled by SDFM has not been established. A fourteen-room 
simulation with fires present in four of the rooms was tested. Using a Pentium II 400 $\mathrm{MHz}$ processor* required $24 \mathrm{~s}$ of time to simulate the $390 \mathrm{~s}$ fire scenario.

The smoke detector algorithms have not yet been tested experimentally and SDFM needs to be tested in buildings that contain real detectors and HVAC systems. There are additional algorithms that need to be added or expanded in SDFM. These algorithms include but are not limited to improving the multiple detector algorithm for a single room, adding lag time algorithms for detectors, expanding the false alarm algorithm, and adding a wall heating algorithm.

Version 1.1 of the SDFM has demonstrated that this type of fire model can give useful results in both simple fire conditions and in fire conditions where flashover occurs.

* The Identification of any commercial product or trade name does not imply endorsement or recommendation by the National Institute of Standards and Technology. 


\section{Appendix A}

A description of each subroutine used in the SDFM is given in this appendix. SDFM is comprised of two distinct parts. The first part is of a data input section, a detector analysis section, a section to call the zone model and a section to evaluate the hazards. The second part is the zone model that is a variant of CFAST. The first part will be designated the sensor-driven model and the second part will be designated the zone model.

\section{Sensor-driven model}

The sensor-driven model is written in FORTRAN 90 and is comprised of the following subroutines and models:

- $\quad$ Pfire

1. Defines variables and allocates space for the arrays,

2. Reads a data file to initialize variables, see appendix B for the input file and a variable list,

3. Initializes variables,

4. Calls subroutines which process sensor signals,

5. If a fire exists, calls subroutines that determine the fire's HRR,

6. If a fire exists, prepares a data file for the zone model and calls the zone model,

7. If a fire exists, calls a subroutine to write an output file identifying the location of fires and rooms that are impacted with heat or smoke.

- Signalc

1. Accepts and interprets signals from the building sensors. If a sensor signal is larger than a user prescribed set point, a fire is detected, for smaller signals but still above the expected ambient signal, a fire is suspected, and for zero signal the detector is labeled as not working.

- Hrrest

1. Converts the analog or digital signal from a sensor to either a temperature or smoke concentration at the detector. Signal lag due to thermal inertia or entry effect is not presently included in the model.

2. Converts temperature or smoke concentration to a HRR.

3. If a temperature sensor, compares the calculated temperature of the upper layer to the estimated sensor temperature. If the sensor temperature is more than $130 \%$ of the upper layer temperature or if the upper layer temperature reaches $500{ }^{\circ} \mathrm{C}$, a fire is assumed to have started in the space.

4. If a smoke sensor, compares calculated smoke concentration in the upper layer to the estimated sensor smoke concentration. If the sensor smoke concentration is more that $130 \%$ of the smoke concentration in the layer, a fire is assumed to have started in the space. 
- Alarm

1. Checks the estimated HRR from each sensor in a room and uses the largest HRR to model the fire in the room.

2. Checks to see if a fire existed during the previous calculation period. If a fire has not been detected in the space, the estimated HRR is compared with a minimum target HRR and if the target HRR is exceeded, a fire is assumed to have started.

3. Retrieves the time from the internal computer clock and provides a fire start time for each room as well as the current time duration of the fire.

4. Calculates the number of rooms that have fires and the time duration to be used in the zone model calculation.

- Heatrr

1. Prepares inputs for the zone model.

- Zonefire

- Initial subroutine for the zone model

- Getvals

1. Retrieves the layer temperatures, height, and room pressure from the zone model.

- Statusr

1. Outputs hazard warnings to the screen based on upper layer temperature and height. Present hazard levels include:

A. Limited visibility warning if the layer height is $2 \mathrm{~m}$ or less

B. Thermal hazard warning if the layer temperature is greater than $50{ }^{\circ} \mathrm{C}$

C. Toxic gas/thermal hazard warning if the layer temperature is greater than 50

${ }^{\circ} \mathrm{C}$ and the layer height is less than $1.5 \mathrm{~m}$

D. Flashover conditions exist if the layer temperature exceeds $500{ }^{\circ} \mathrm{C}$

- Plumezero

1. Calculates the heat release rate of the fire source when there is a hot upper layer present.

- Detradius

1. Provides a default location of the detector with respect to the fire when only a single detector is available in a fire room.

- Pdata

1. Defines global variables for the calculation of heat release rate of the fire in the presence of a layer.

- Dfzero

1. Double precision nonlinear equation root finder. 


\section{Zone model}

The zone model used is a variant of CFAST version 3.1.6 and is written in FORTRAN 90. A primary design feature of this model is that it must be able to handle a building with a large number of rooms and complete a calculation cycle faster than real time. The model therefore contains only the essential physics necessary to follow the fire development. The differential equations solved by the model include the pressure equation, the temperature for each layer, the volume of each layer and the oxygen content for each layer. As the model evolves, additional physics such as the carbon monoxide concentration found in the layer will likely be added. The subset of physics included in the model is:

- Flow of gases through vents,

- Radiation loss to the walls but no wall heating,

- Convective heat loss to the walls but no wall heating,

- HVAC flow

The calculation of wall heating is time intensive. The present algorithm maintains the walls at a constant temperature. This assumption is satisfactory early in the fire. It is planned to add wall heating to the model in order to predict structural failure based on wall temperature and time of exposure.

Subroutines and modules included in the zone model are:

- Convec

1. Calculates convective heat flux to a wall segment.

- Convecflow

1. Calculates convective heat flow to the walls, ceiling and floor for each room.

- Datacopy

1. Copies global variables into specific variables compatible with the solver ddassl.

- Ddassl

1. Double precision differential equation/algebraic equation solver.

- Fireflow

1. Set of subroutines that calculates entrainment for each fire.

- Getdp

1. Calculates pressure difference across a vent slab.

- hvacflow

1. Set of subroutines that calculate flow in a room due to HVAC systems.

- Initmm

1. Set of subroutines that initialize the zone model.

- Interp

1. Provides an interpolation of a variable between two time steps.

- Precision

1. Sets the model calculations to double precision.

- Readdata

1. Subroutines that reads and writes data files. 
- Resid

1. Used by the solver ddassl to provide the right hand side of the differential equations.

- Solve

1. Provides the solution structure for the zone model.

- Ventflow

1. Calculates flow of gas through vents.

- Zonedata

1. Modules that define the global variables used by the zone model and allocate space used by the arrays.

- Zonefire

1. Called by the SDFM.

2. Provides calls to the subroutines that initialize the zone model.

3. Calls the subroutine Solve that starts the solution phase of the zone model. 


\section{Appendix B}

The sensor-driven fire model may use up to three input data files to initialize the model and to enter sensor information. When the internal computer clock is supplying the time or the time is provided with the sensor signals, only a single data file is required. The first step in constructing this file is to make a drawing of the structure to be modeled showing room dimensions and connectivity between the rooms. Each room should be numbered on the drawing starting with the number 1 . The data file is then constructed using the following data fields by line:

1. Number of rooms, number of detectors, prediction interval, number of vents, type of file.

2. Detector: type, set-point, ambient signal, $x$ location, y location, room location for the detector, calibration constants: $a, b$, c.

3. Room: length, width, ceiling height, floor height, ambient temperature, lower lefthand corner of room: $\mathrm{x}, \mathrm{y}$. The computer automatically generates room numbers based on the sequence of room dimensions entered in during this step. Room number 1 on the drawing should coincide with the first set of dimensions entered in here.

4. Vent flow direction is given as two room numbers: from room, to room. The flow will go in either direction.

5. Vent dimensions: sill height, soffit height, width of vent, vent offset.

For line 1 inputs:

- The number of rooms value should include all spaces such as halls, closets and stairways.

- More than one detector can be located in a single room but not every room requires a detector. The number of detectors may be either greater than or less than the number of rooms.

- The prediction interval is used to estimate the fire growth and is the number of data points used in the linear extrapolation of the HRR for a given fire room.

- Any number of vents may be located at the walls of a particular room and may connect to any room in the structure. The outside is represented by a room number of 0 .

- Ceiling vents and floor vents may be implemented by offsetting the floor heights of the rooms such that a wall vent either at the ceiling or at the floor can be used to connect the two rooms.

- The type of file entry will be 1, 2 or 3 depending on the type of input file. File type 1 uses a sensor input file that contains only sensor signals with the time taken from the internal computer clock. File type 2 is a sensor file that includes both the time and sensor data. File type 3 is used in conjunction with the Virtual Cybernetic Building Test-bed (VCBT) and contains sensor data and times generated by the VCBT.

For line 2 inputs:

- Detector type is 1 for a heat detector and 2 for a smoke detector. 
- Set-point is the user defined sensor signal level that triggers an alarm.

- Ambient signal is the signal that the sensor would send to the panel in a no fire scenario. This input will eventually be overwritten when a history file for the detector is established.

- The $\mathrm{x}$ and $\mathrm{y}$ locations for the detector is the position of the sensor in meters from the lower left-hand corner of the room as viewed from above.

- The room location is the room number where the detector is located.

- The sensor constants $\mathrm{a}, \mathrm{b}$, and $\mathrm{c}$ refer to the coefficients of the equation $\mathrm{ax}^{2}+\mathrm{bx}+\mathrm{c}$. The equation is used to convert a detector signal to temperature, smoke concentration, etc.

For line 3 inputs:

- Length, width, ceiling height and floor height are in meters. The floor height is a reference height with respect to ground level or lowest elevation of the building.

- Ambient temperature of each room in Kelvin.

- The position of lower left-hand corner of the room as viewed from above with respect to the lower left-hand corner of the building in meters. The position is entered as $\mathrm{x}, \mathrm{y}$. A right-handed coordinate system is used with up being the $\mathrm{z}$ direction.

For line 4 inputs:

- From room and to room are room numbers of rooms generated by the sequence of entries in line 3. The rooms do not have to be adjacent or even on the same floor. Room number zero represents the outside which is at ambient temperature and pressure. This input establishes the connectivity of flow for a particular vent.

For Line 5 inputs:

- Sill height is the distance in meters from the floor to the bottom of the vent.

- Soffit height is the distance in meters from the floor to the top of the vent.

- The width of the vent is in meters.

- Vent offset is the distance that the edge of the vent is located with respect to the lefthand side of the wall as you face the wall.

When the model is operated in the VCBT environment, the subroutine pfire is called by the VCBT and passed three arrays vcbsig, vcbalarm, and vcbroom. The array vcbsig contains a 1 or 2 in the first postion to indicate whether pfire is being called for the first time (1) or for a subsequent time (2). The second postion in the array is the time and the subsequent positions are the sensor signals. Vcbalarm and vcbroom are arrays that pass information back to the VCBT. The array vcbalarm has a length equal to the number of detectors. A value of 1 for each detector means no fire while a value of 2 means a fire. The array vcbroom has a length equal to the number of rooms. This array carries the hazard warning information for each room with the value: 1 no hazard, 2 limited visibility, 3 toxic gas/thermal hazard and 4 flashover conditions exist. 


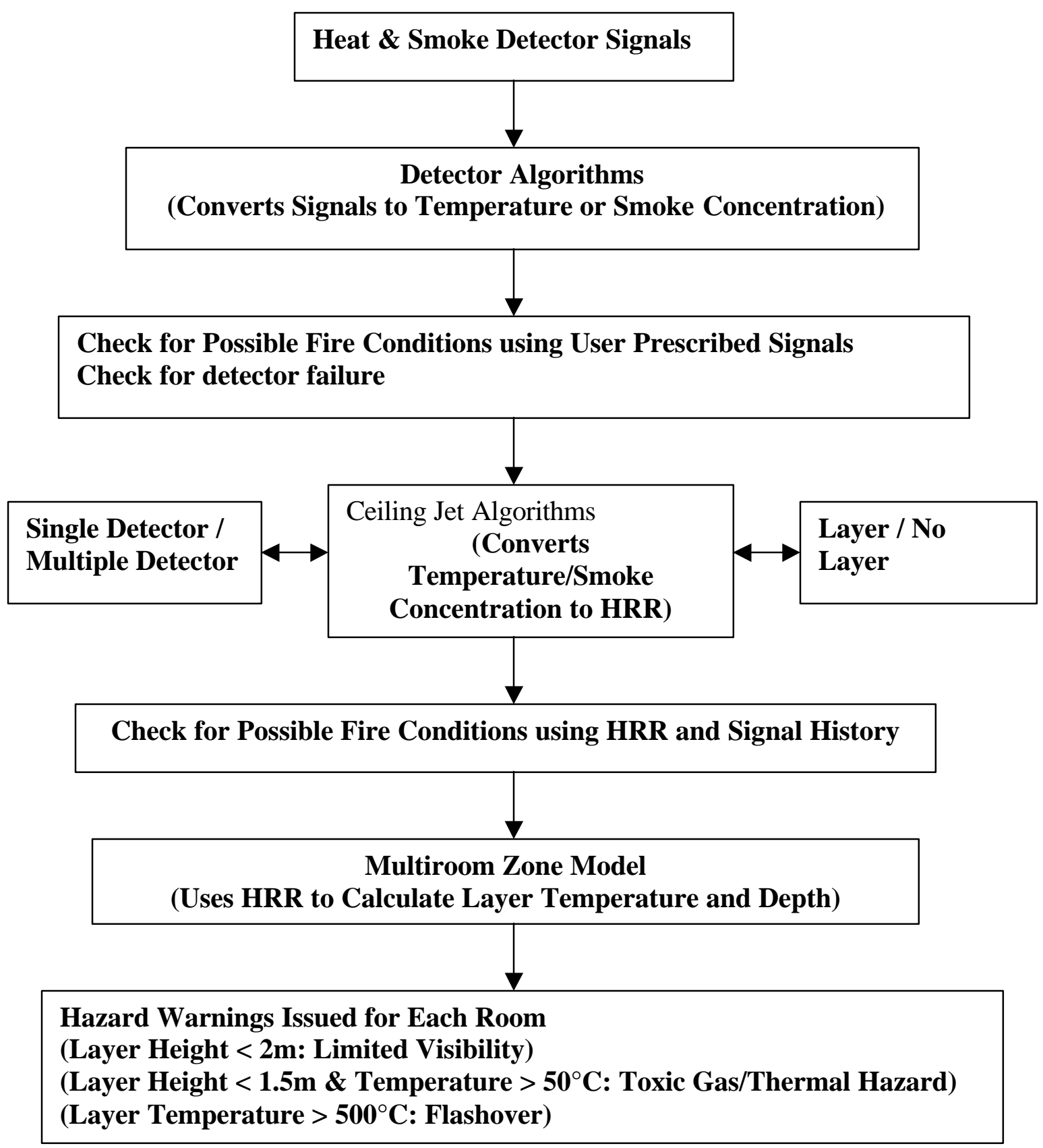

Figure 1 Flow Chart for SDFM Version 1.1 


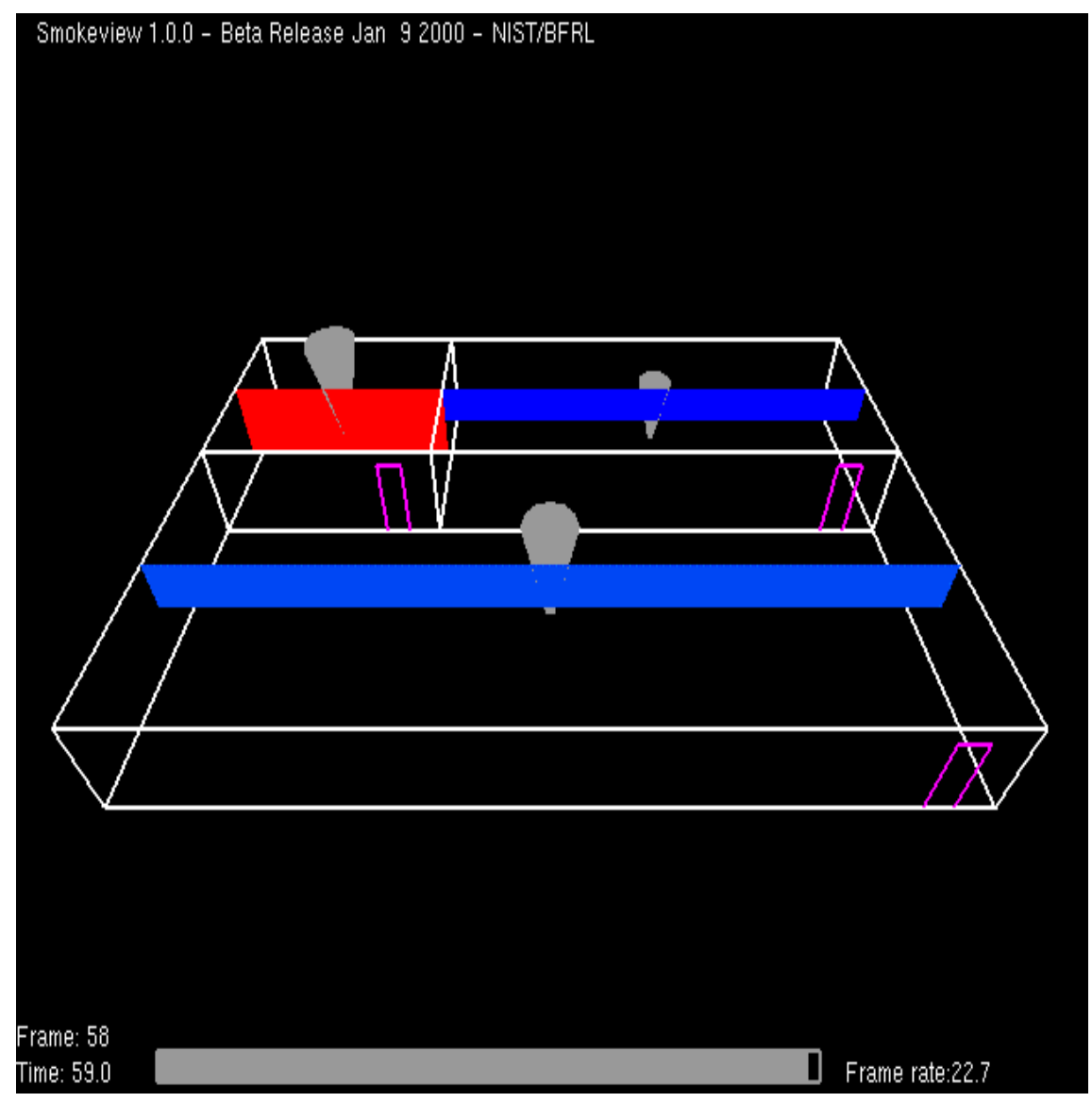

Figure 2 Three-room VCBT simulation using SDFM and displayed using Smokeview. The layer temperature and depth are represented by the colored rectangles. The HRR of each fire is indicated by the size of the inverted cones. 


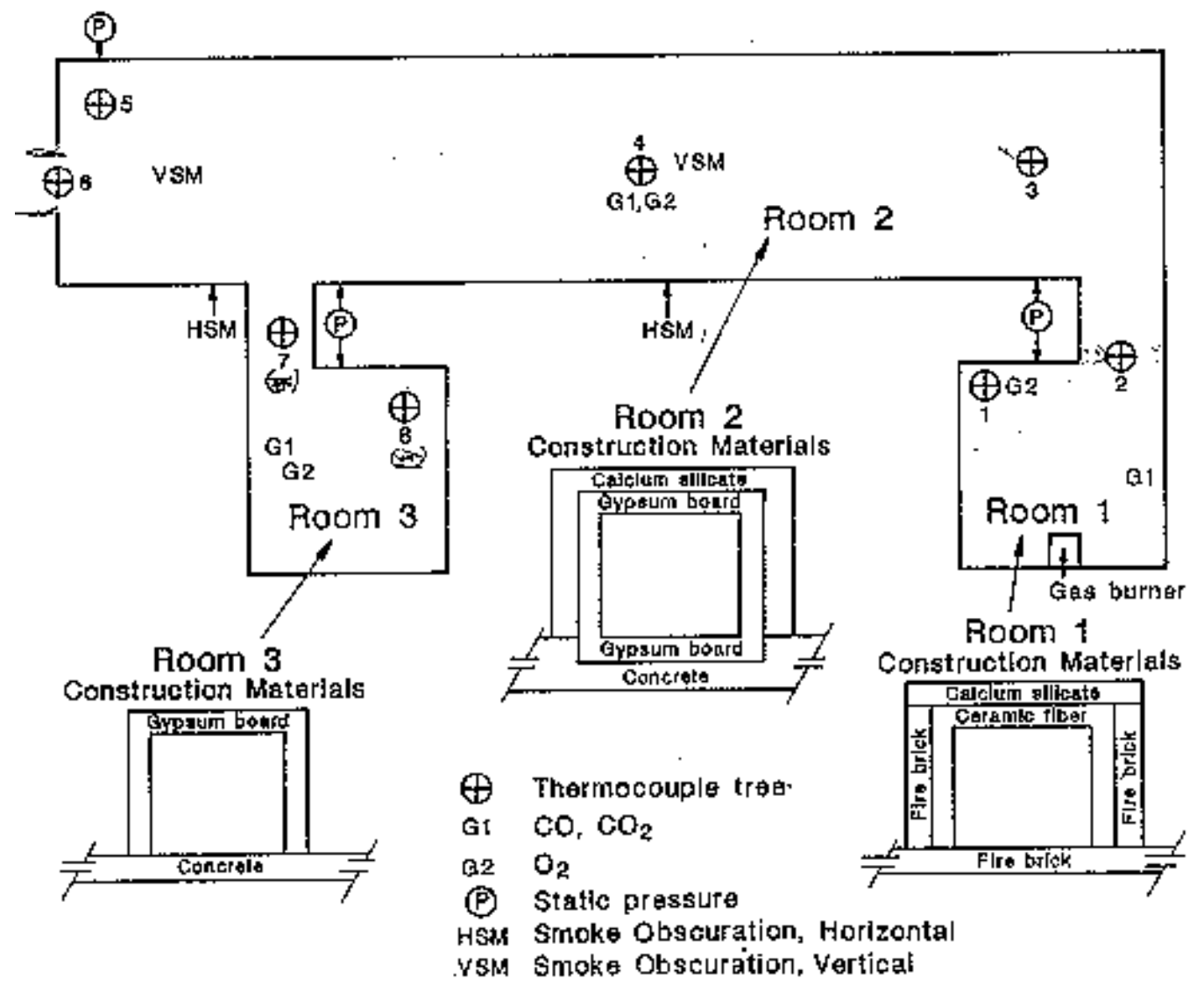

Figure 3 Three-room fire experiment. The fire is in room 1. Thermocouples are shown by pluses enclosed by circles. 


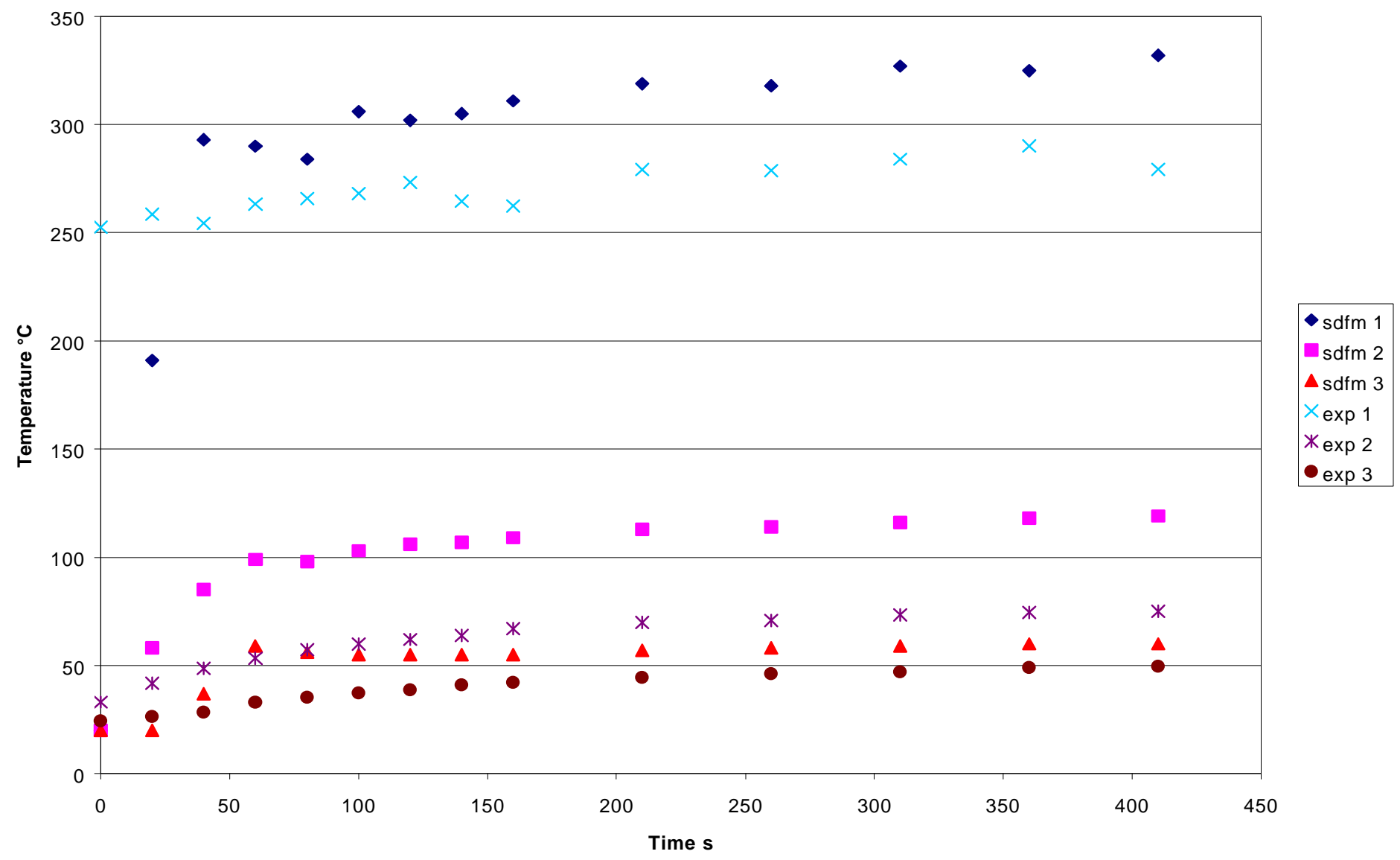

Figure 4 Comparison of predicted and measured upper layer temperature for the three room experiments. Predictions of the SDFM are labeled "sdfm" with the room number while the measurements are labeled "exp" with the room number. 


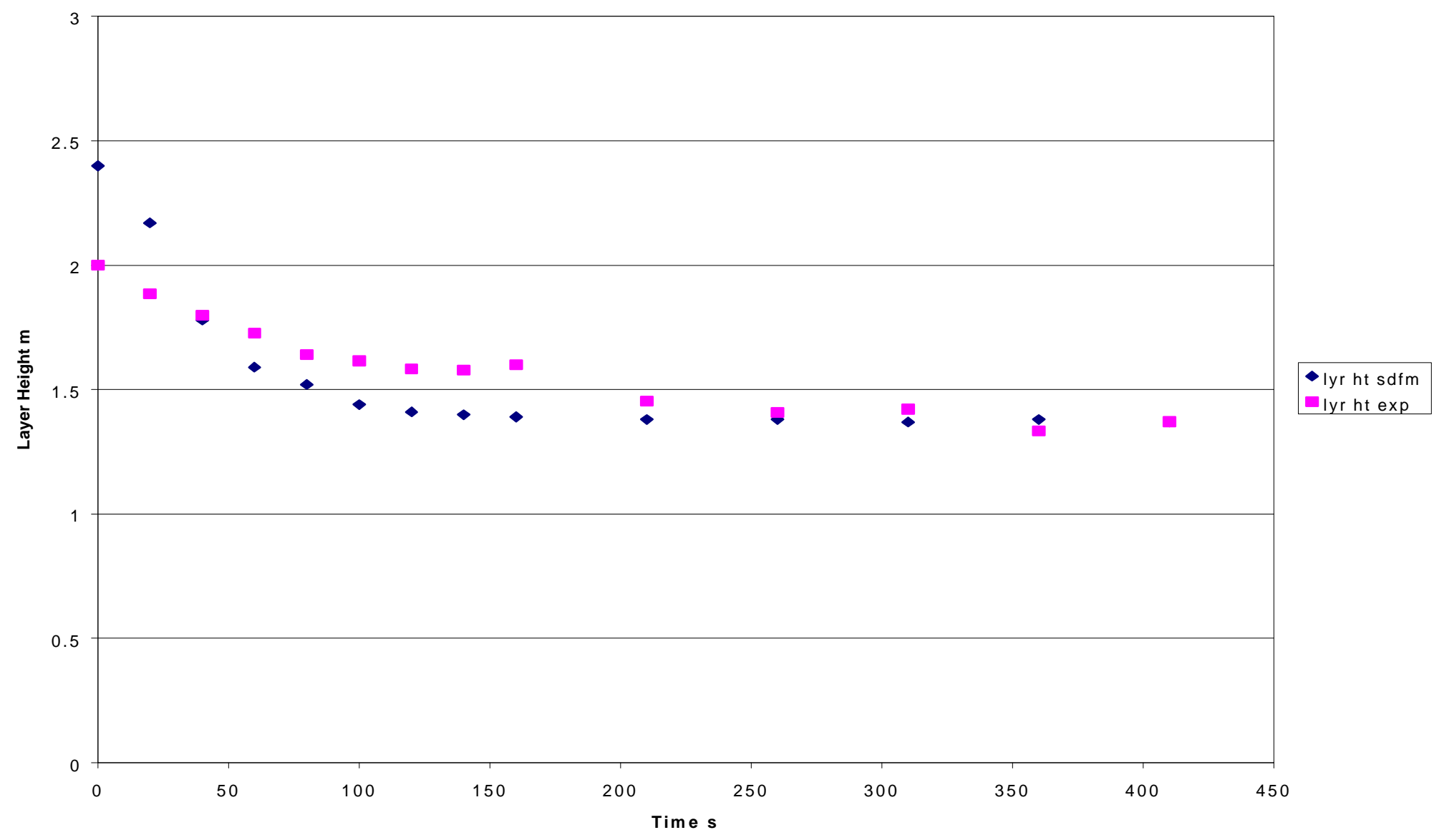

Figure 5 Predicted and measured layer heights for room 2 of the three-room experiment. Predictions of the SDFM are labeled "lyr ht sdfm" while the measurements are labeled "lyr ht exp". 

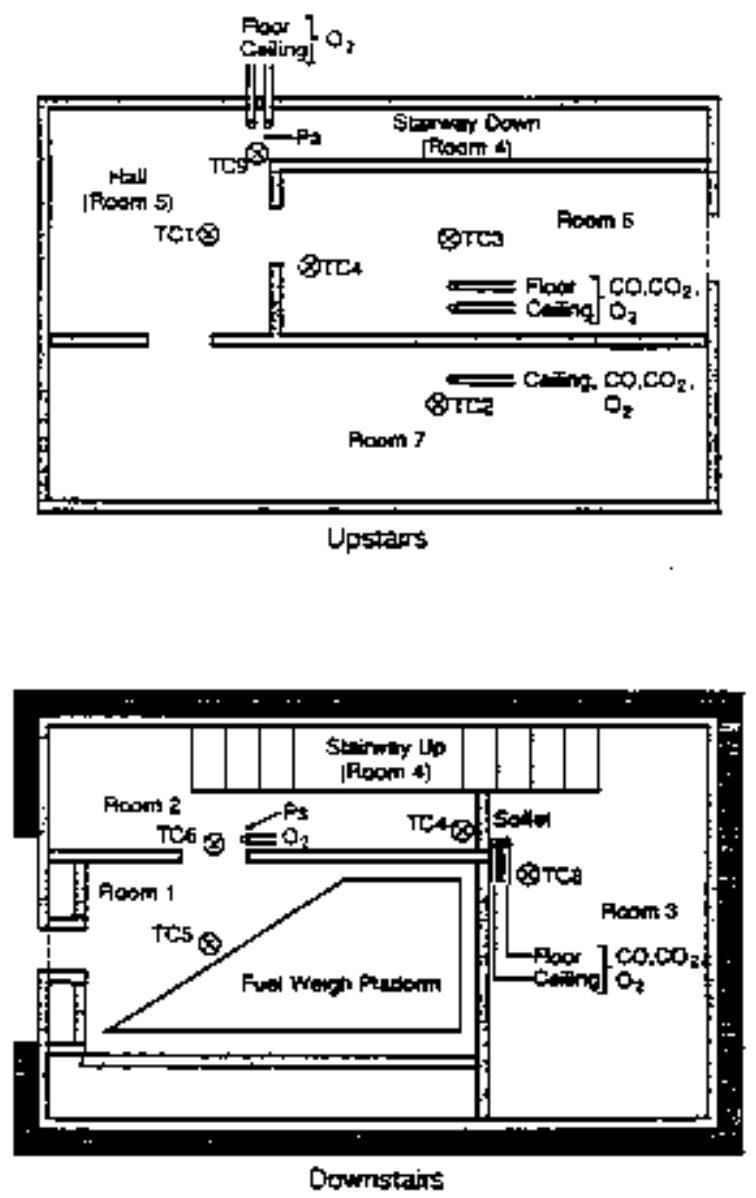

Figure 6 Plan view of the Sharon 2 townhouse. The fire is in room 1 and the thermocouples are indicated by TCX. 


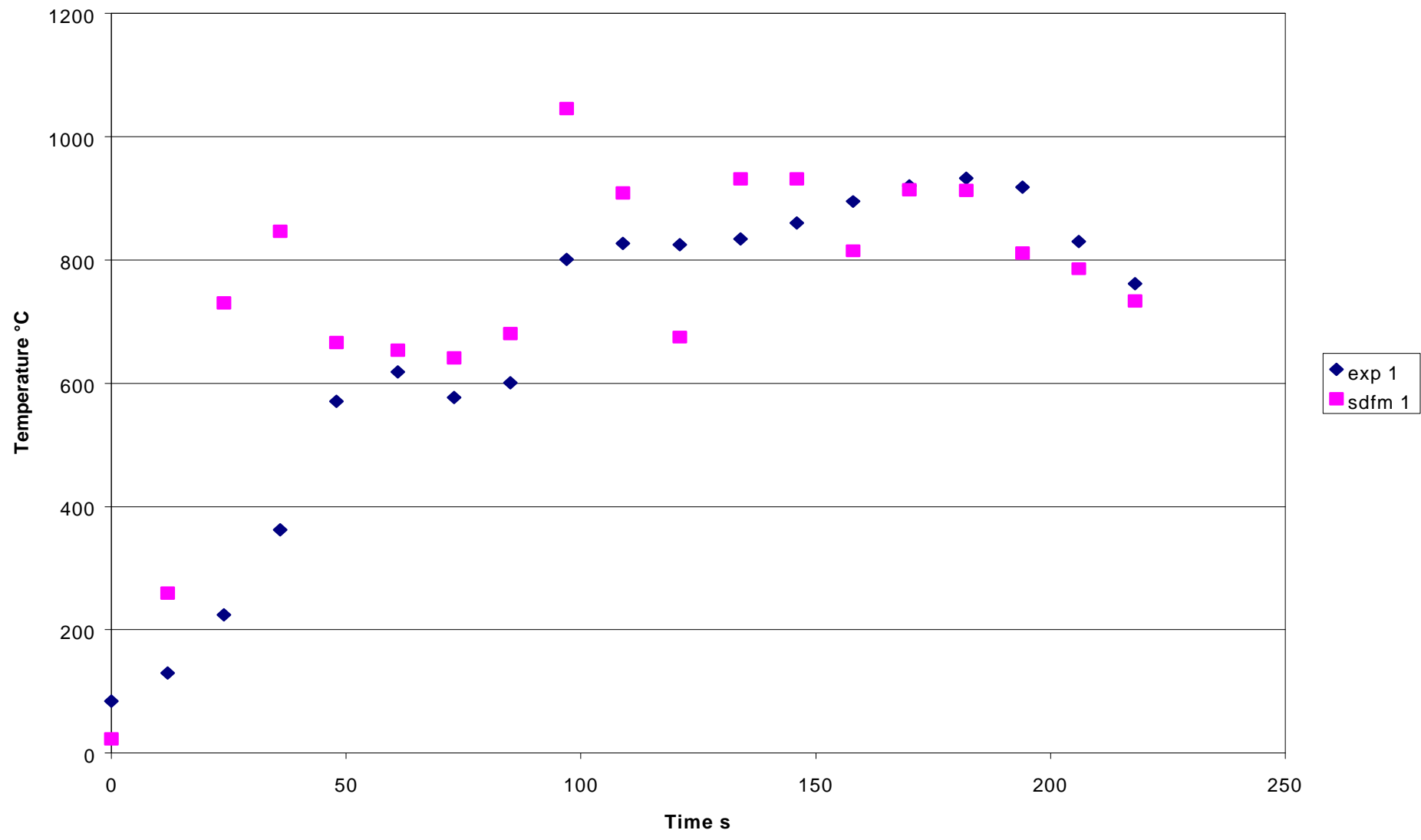

Figure 7 Sharon 2 burn room comparison of predicted and measured upper layer temperature. Predictions of the SDFM are labeled "sdfm" while the measurements are labeled "exp". 


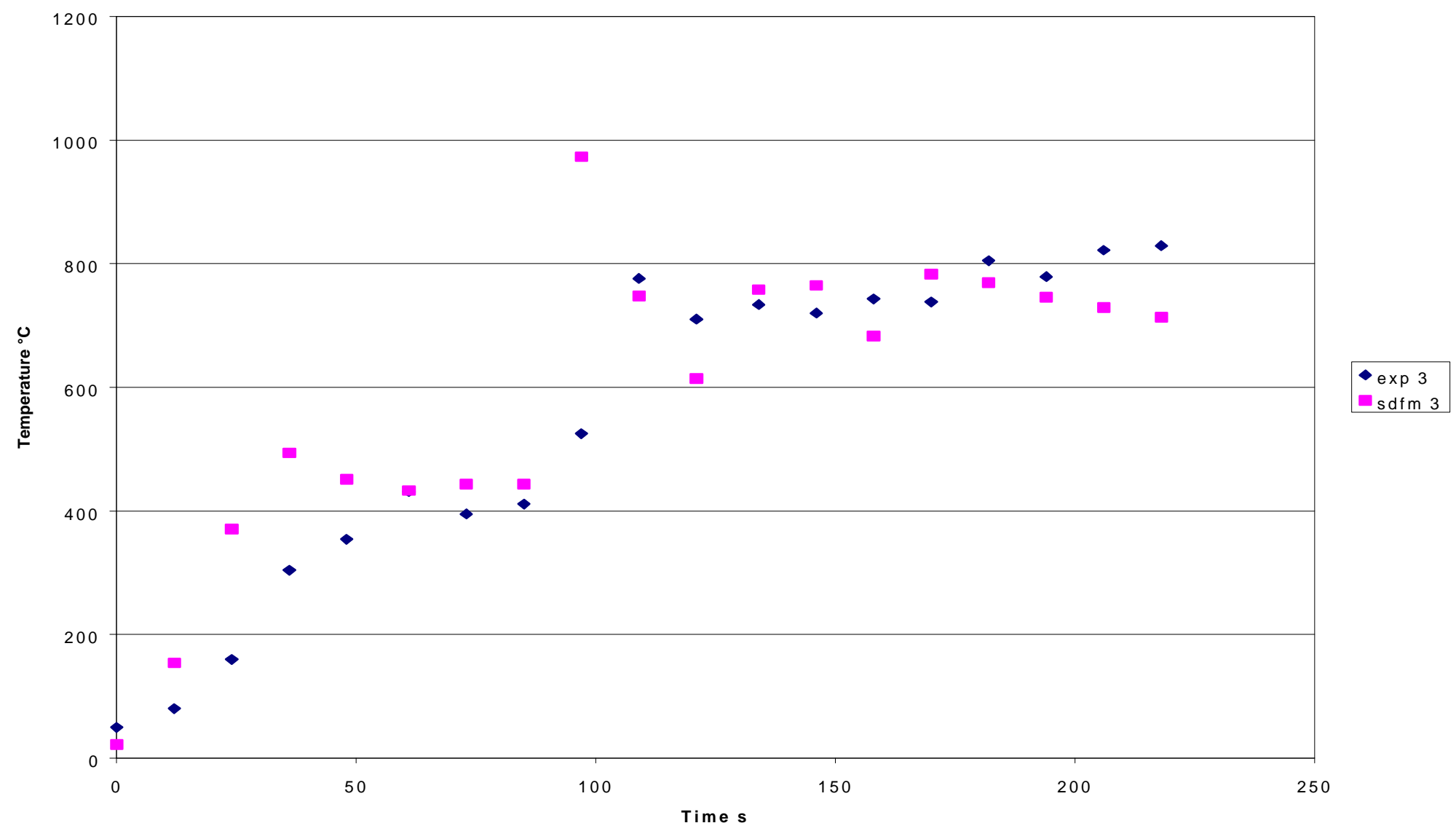

Figure 8 Sharon 2 lower level hallway comparison of predicted and measured upper layer temperature. Predictions of the SDFM are labeled "sdfm" while the measurements are labeled "exp". 


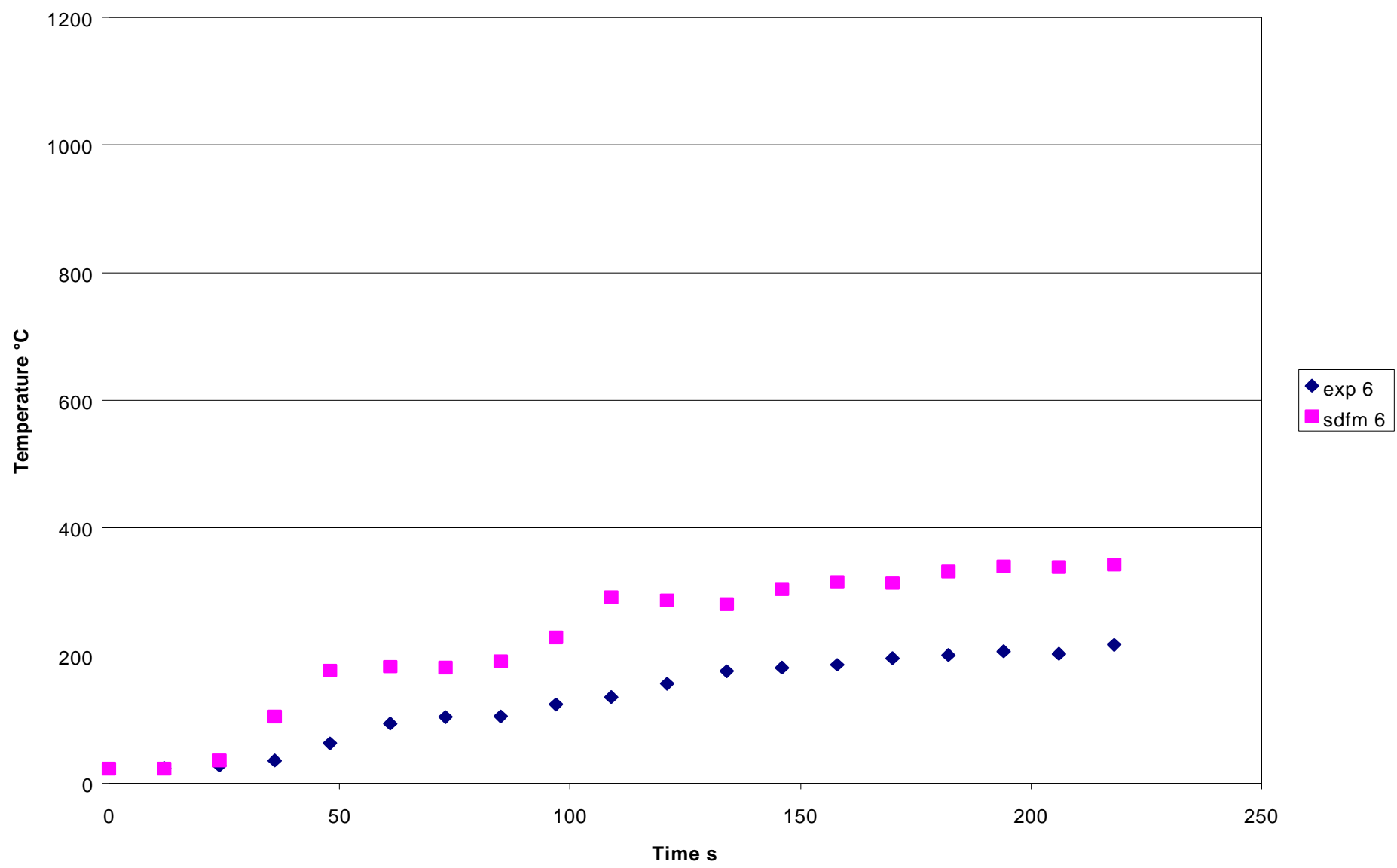

Figure 9 Sharon 2 upper hallway comparison of predicted and measured upper layer temperature. Predictions of the SDFM are labeled "sdfm" while the measurements are labeled "exp". 


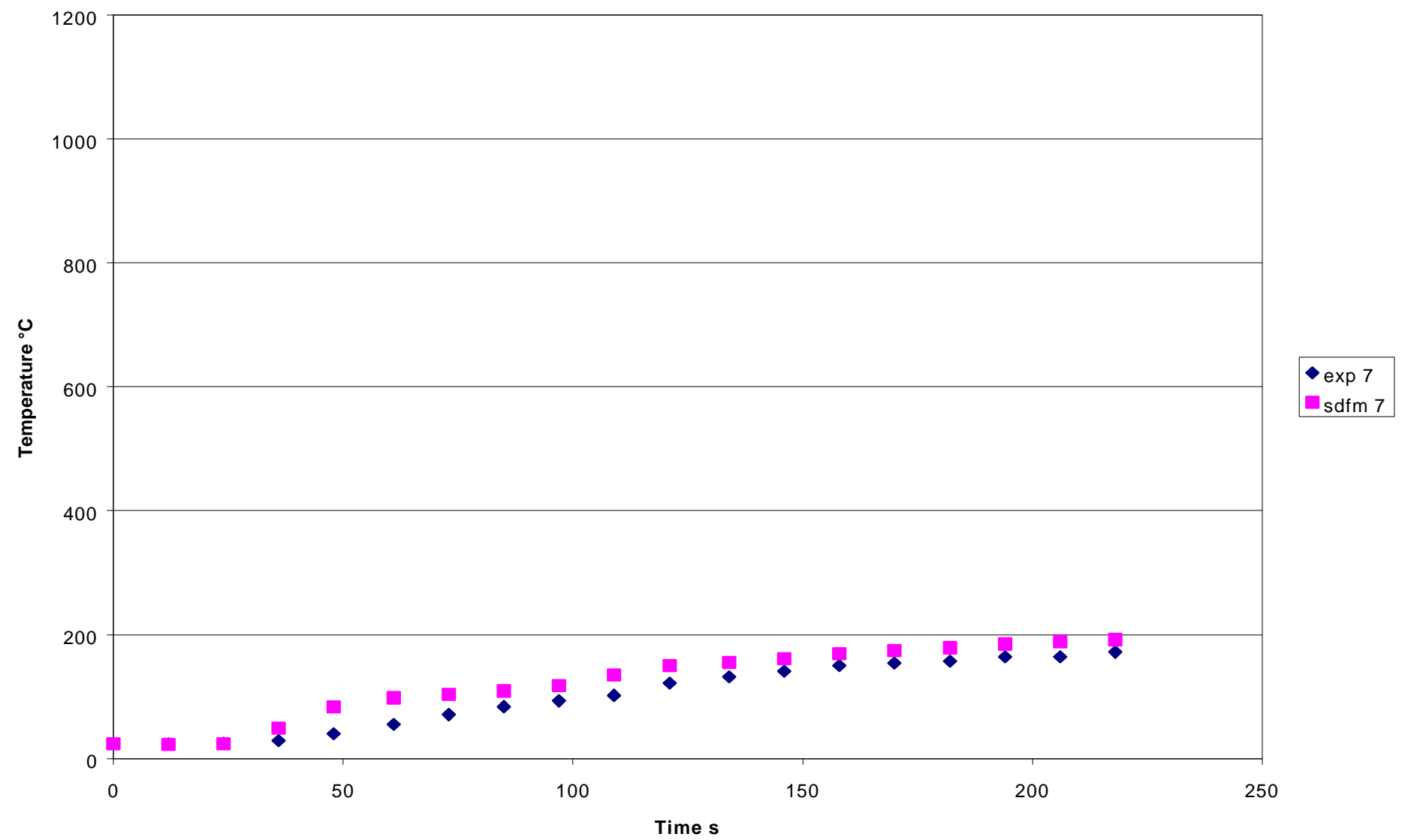

Figure 10 Sharon 2 upper level south bedroom comparison of predicted and measured upper layer temperature. Predictions of the SDFM are labeled "sdfm" while the measurements are labeled "exp". 


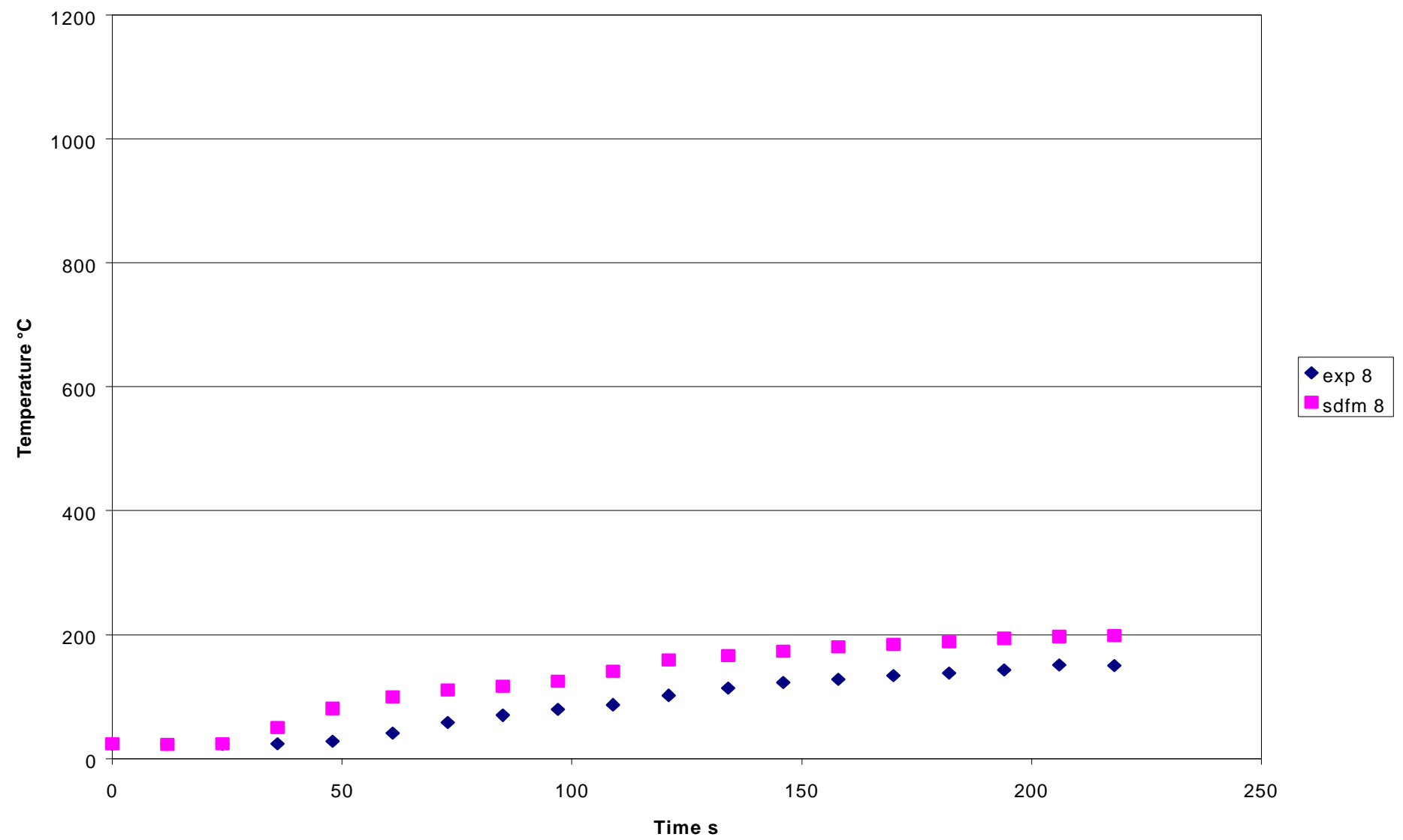

Figure 11 Sharon 2 upper level north bedroom comparison of predicted and measured upper layer temperature. Predictions of the SDFM are labeled "sdfm" while the measurements are labeled "exp". 


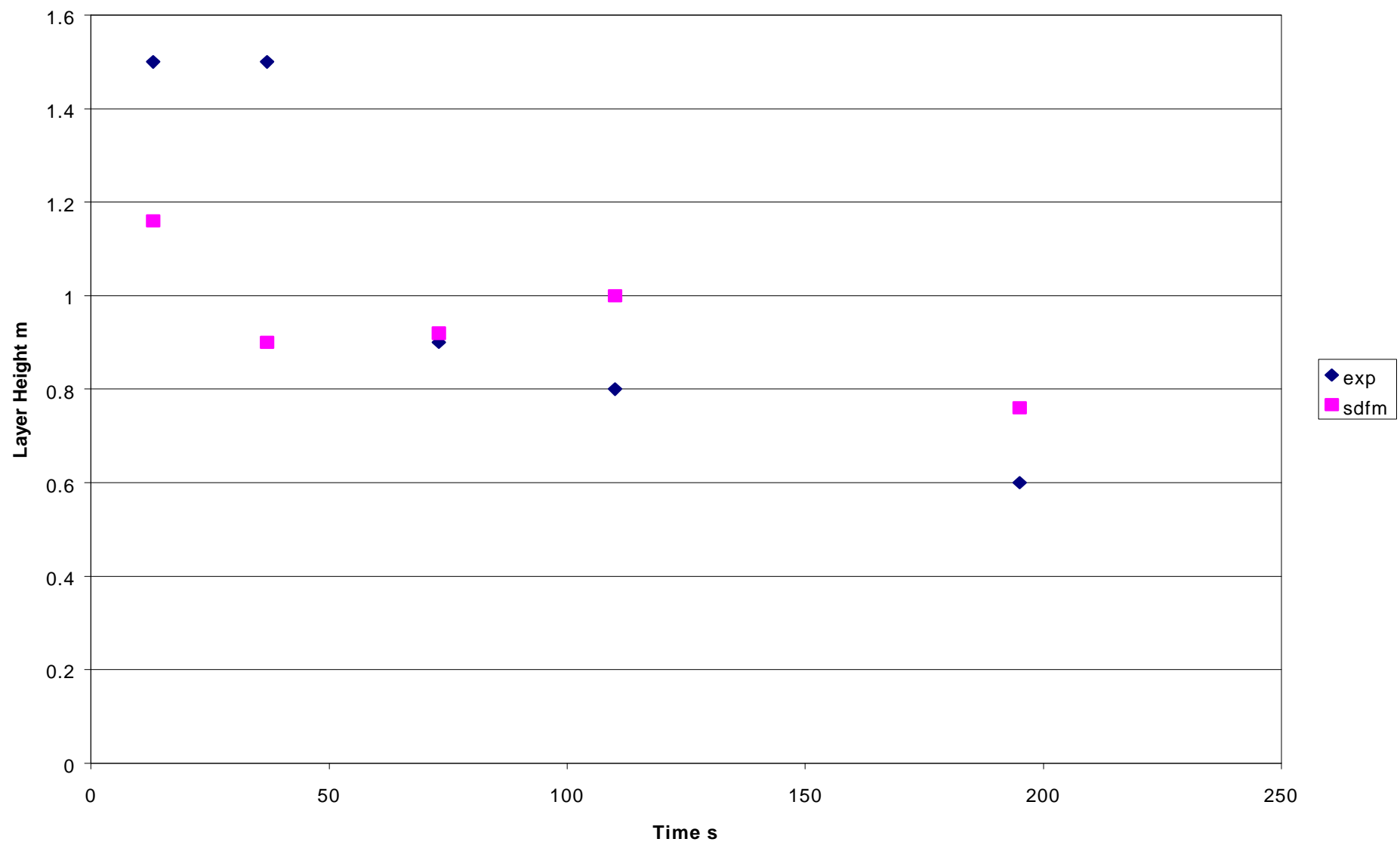

Figure 12 Sharon 2 burn room comparison of predicted and measured upper layer heights. Predictions of the SDFM are labeled "sdfm" while the measurements are labeled "exp". 


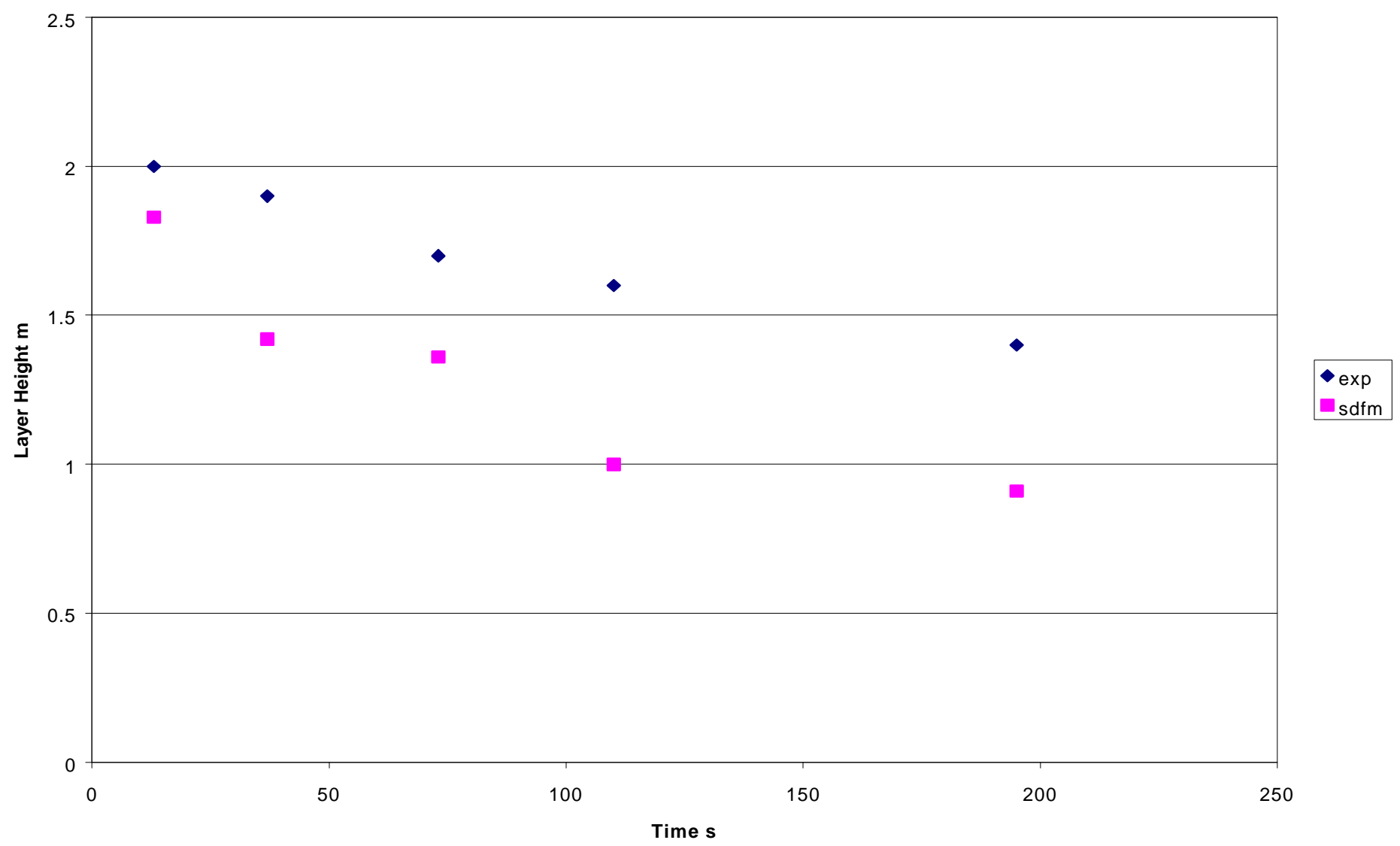

Figure 13 Sharon 2 lower hall comparison of predicted and measured layer height. Predictions of the SDFM are labeled "sdfm" while the measurements are labeled "exp". 


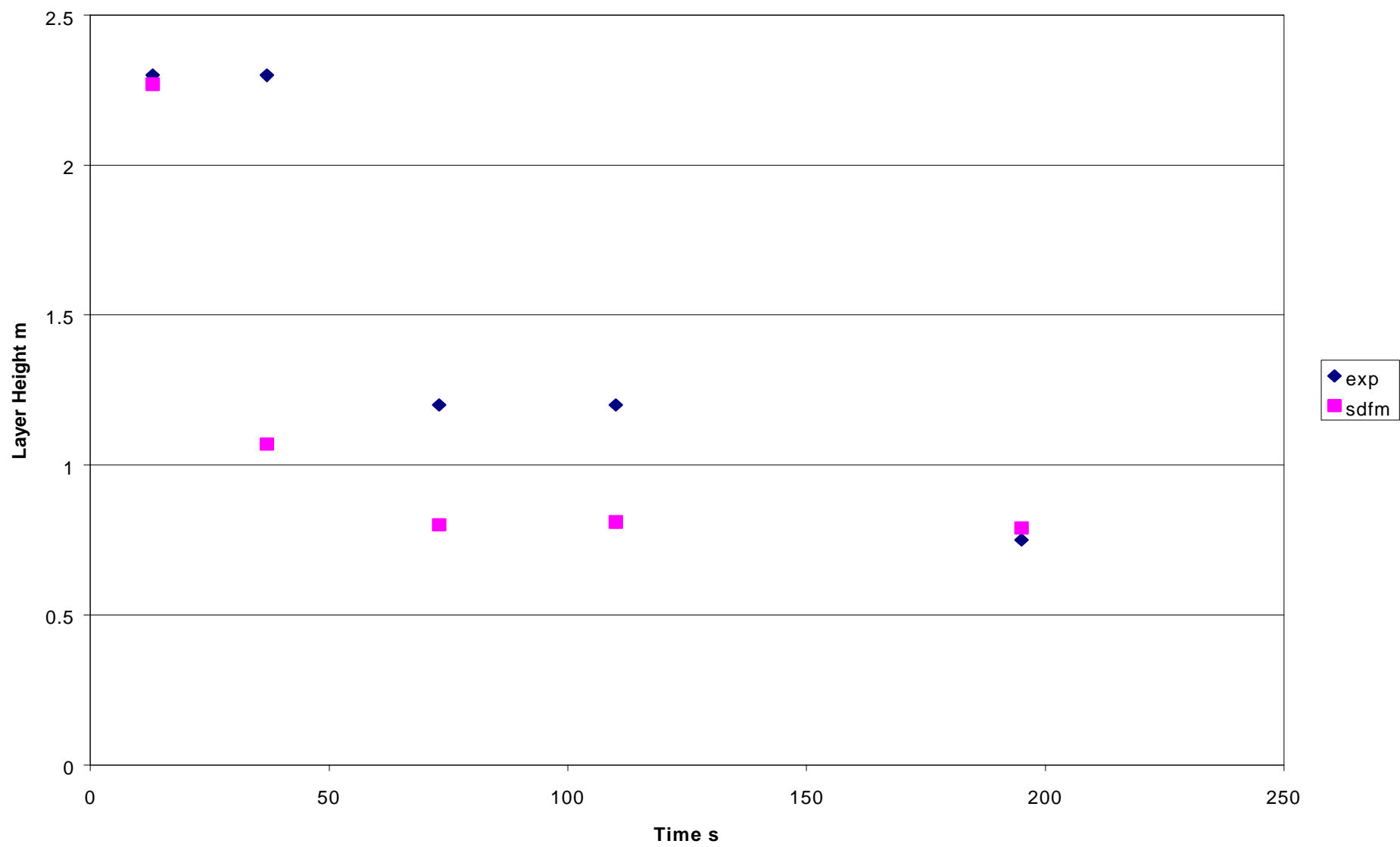

Figure 14 Sharon 2 upper level hallway comparison of predicted and measured layer height. Predictions of the SDFM are labeled "sdfm" while the measurements are labeled "exp". 


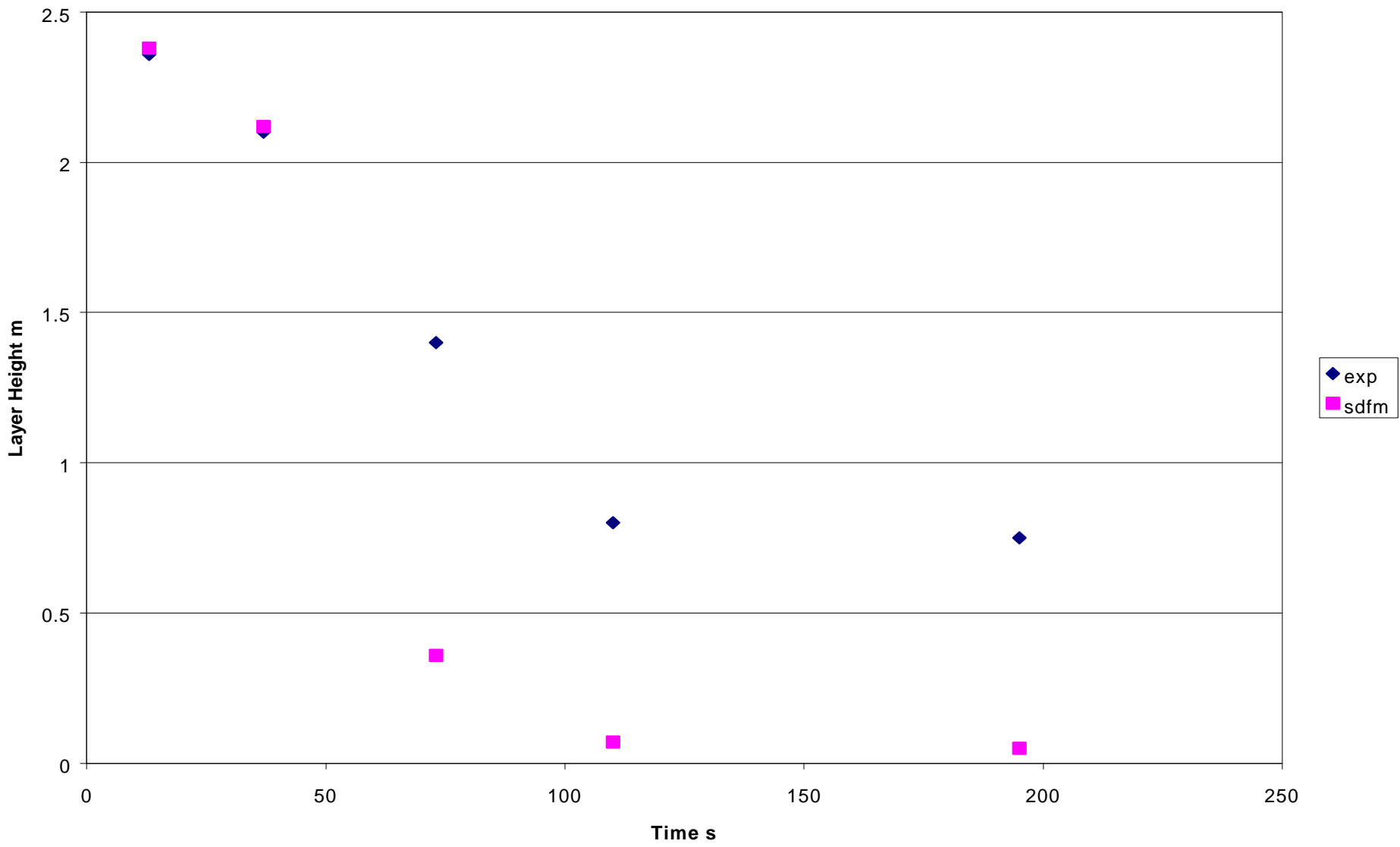

Figure 15 Sharon 2 upper level north bedroom comparison of predicted and measured upper layer height. Predictions of the SDFM are labeled "sdfm" while the measurements are labeled "exp". 


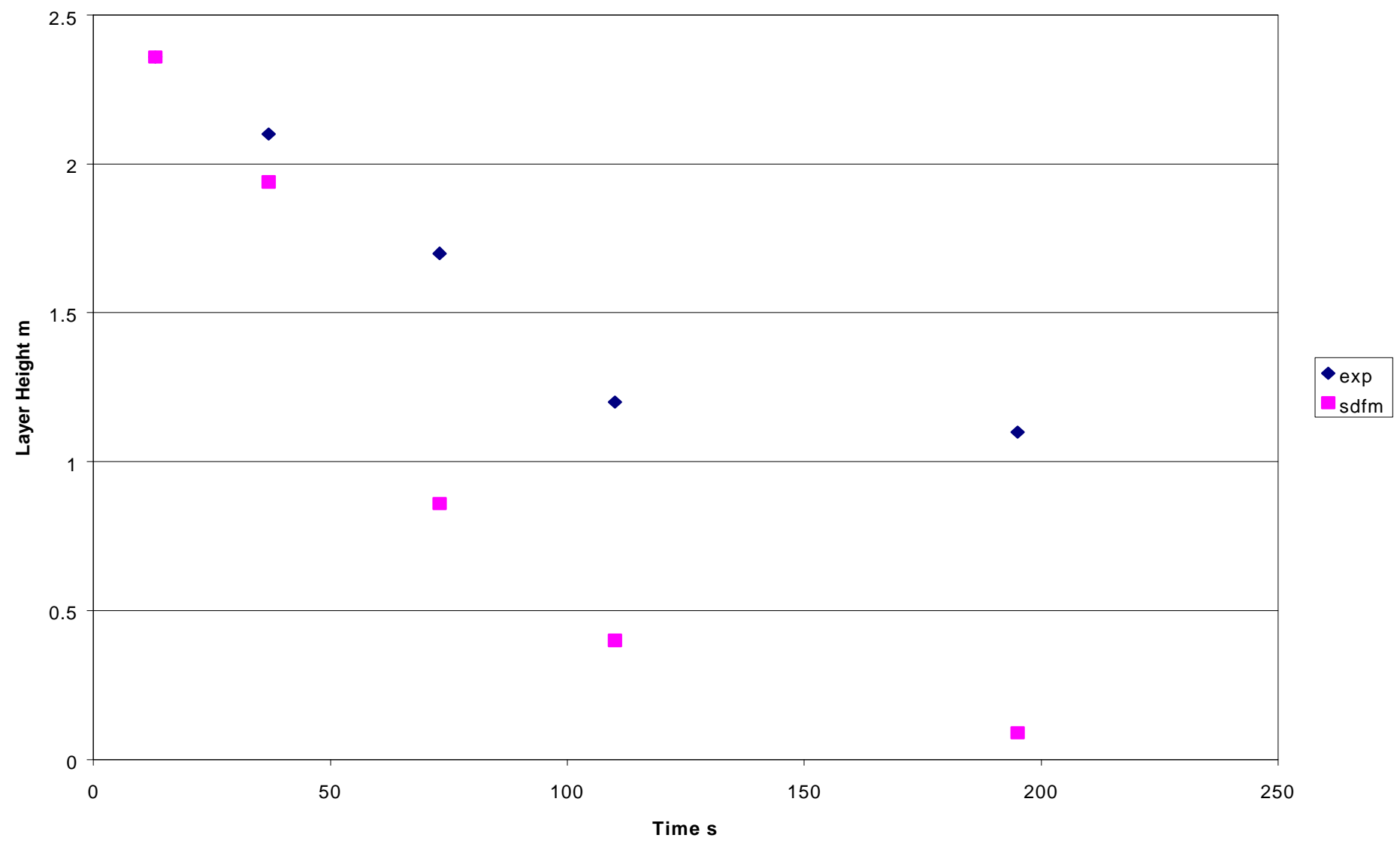

Figure 16 Sharon 2 upper level south bedroom comparison of predicted and measured layer height. Predictions of the SDFM are labeled "sdfm" while the measurements are labeled "exp". 
${ }^{i}$ Nelson, H. E., "Functional Programming/Research Planning for High Technology Federal Office buildings," National Institute of Standards and Technology NBSIR 842828, (1984) p. 28.

ii Davis, W. D., "The Zone Fire Model Jet: A Model for the Prediction of Detector Activation and Gas Temperature in the Presence of a Smoke Layer," National Institute of Standards and Technology, NISTIR 6324, (1999) pp. 1-51.

${ }^{\text {iii }}$ Yang, J. C., Hamins, A., and Kashiwagi, T., " Estimate of the Effect of Scale on Radiative Heat Loss Fraction and Combustion Efficiency, " Combustion Science and Technology, 96, (1994) pp. 183-188.

iv Evans, D. D., "Calculating Sprinkler Actuation Time in Compartments," Fire Safety J., 9, 1985, pp. 147-155.

' Davis, W. D., and Reneke, P., "Predicting Smoke Concentration in the Ceiling Jet", National Institute of Standards and Technology, NISTIR 6480, (2000) pp. 1-12.

vi Heskestad, G., "Fire Plumes," The SFPE Handbook of Fire Protection Engineering, 1992, p. 1-112.

${ }^{\text {vii }}$ Peacock, R. D., Forney, G. P., Reneke, P., Portier, R., and Jones, W. W., "CFAST, the Consolidated Model of Fire Growth and Smoke Transport," National Institute of Standards and Technology, NISTTN 1299, (1993) pp. 1-235.

viii McGrattan, K. B. and Forney, G. P., "Fire Dynamics Simulator - User's Manual," National Institute of Standards and Technology, NISTIR 6469, (2000) pp.1-49.

${ }^{\text {ix }}$ Forney, G. P. and McGrattan, K. B., "User's Guide for Smokeview Version 1.0 - A Tool For Visulalizing Fire Dynamics Simulation Data," National Institute of Standards and Technology, NISTIR 6513, (2000) pp. 1-32.

${ }^{x}$ Peacock, R. D., Davis, S., and Lee, B. T., "An Experimental Data Set for the Accuracy Assessment of Room Fire Models," National Institute of Standards and Technology, NBSIR 88-3752, (1988) pp. 111.

${ }^{x i}$ Levine, R. S. and Nelson, H. E., "Full Scale Simulation of a Fatal Fire and Comparison of Results with Two Multiroom Models Volume II - DATA," National Institute of Standards and Technology, NISTIR 90-4268, (1990) pp. 1-125. 